NISTIR 7880-34

\title{
NIST Micronutrients Measurement Quality Assurance Program Winter, Spring, and Fall 1990 Comparability Studies
}

Results for Round Robins XVIII, XIX, and XX Fat-Soluble Vitamins and Carotenoids in Human Serum

Neal E. Craft (Former Employee)

David L. Duewer

Margaret C. Kline

Sam A. Margolis (Retired)

Willie E. May

Katherine E. Sharpless

Jeanice B. Thomas

This publication is available free of charge from http://dx.doi.org/10.6028/NIST.IR.7880-34 
NISTIR 7880-34

\title{
NIST Micronutrients Measurement Quality Assurance Program Winter, Spring, and Fall 1990 Comparability Studies
}

\author{
Results for Round Robins XVIII, XIX, and XX \\ Fat-Soluble Vitamins and Carotenoids in Human Serum
}

Neal E. Craft (Former Employee)

David L. Duewer

Sam A. Margolis (Retired)

Jeanice B. Thomas

Chemical Sciences Division, Materials Measurement Laboratory

Margaret C. Kline

Biomolecular Measurement Division, Materials Measurement Laboratory

Katherine E. Sharpless

Office of Special Programs

Wille E. May

Under Secretary of Commerce for Standards and Technology and Director

This publication is available free of charge from

http://dx.doi.org/10.6028/NIST.IR.7880-34

June 2015

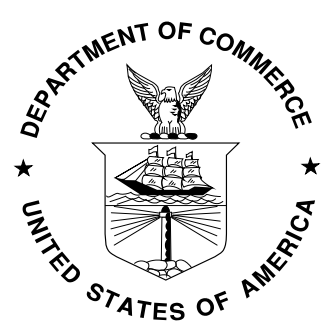

U.S. Department of Commerce

Penny Pritzker, Secretary 
(This page intentionally blank) 


\begin{abstract}
The National Institute of Standards and Technology coordinates the Micronutrients Measurement Quality Assurance Program (MMQAP) for laboratories that measure fat- and water-soluble vitamins and carotenoids in human serum and plasma. This report describes the design of and results for the Winter, Spring and Fall 1990 MMQAP measurement comparability improvement studies: 1) Round Robin XVIII Fat-Soluble Vitamins and Carotenoids in Human Serum, 2) Round Robin XIX FatSoluble Vitamins and Carotenoids in Human Serum, and 3) Round Robin XX Fat-Soluble Vitamins and Carotenoids in Human Serum. The materials for Round Robin XVIII were shipped to participants in January 1990; participants were requested to provide their measurement results by March 5, 1990. The materials for Round Robin XIX were shipped to participants in May 1990; participants were requested to provide their measurement results by July 6, 1990. The materials for Round Robin XX were shipped to participants in August 1990; participants were requested to provide their measurement results by October 22, 1990.
\end{abstract}

\title{
Keywords
}

Human Serum

Retinol, $\alpha$-Tocopherol, Total and Trans- $\beta$-Carotene 


\section{Table of Contents}

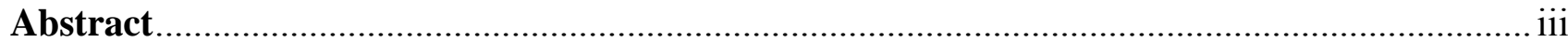

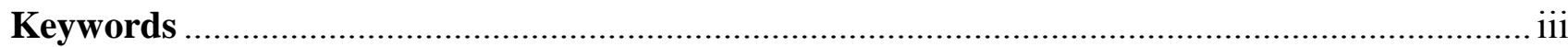

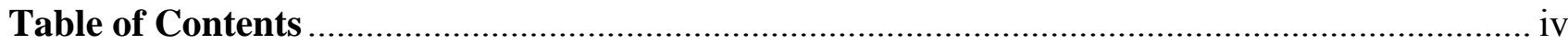

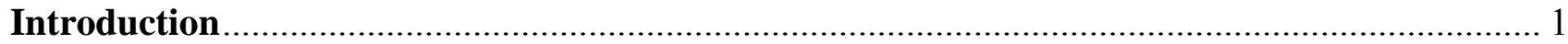

Round Robin XVIII: Fat-Soluble Vitamins and Carotenoids in Human Serum ..................... 1

Round Robin XIX: Fat-Soluble Vitamins and Carotenoids in Human Serum.......................... 2

Round Robin XX: Fat-Soluble Vitamins and Carotenoids in Human Serum ........................... 2

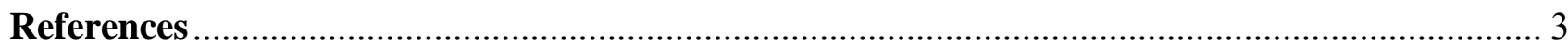

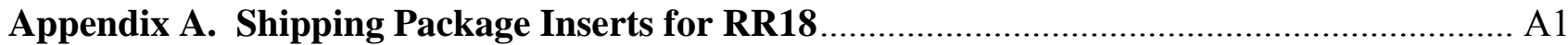

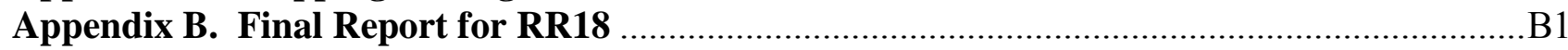

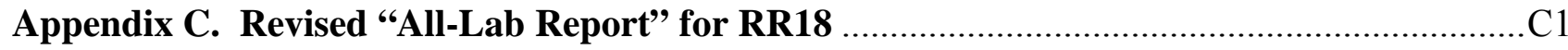

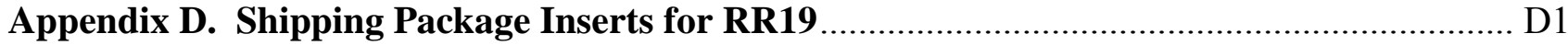

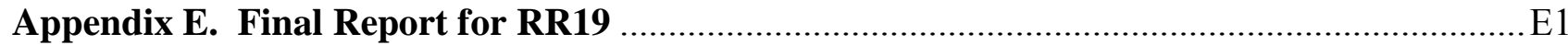

Appendix F. Revised “All-Lab Report” for RR19 .......................................................F1

Appendix G. Shipping Package Inserts for RR20 .......................................................... G1

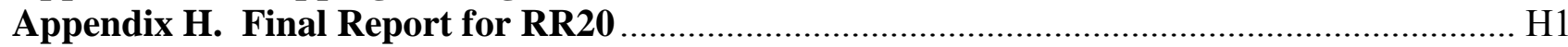

Appendix I. Revised “All-Lab Report” for RR20 ............................................................ I1 


\section{Introduction}

Beginning in 1988, the National Institute of Standards and Technology (NIST) has coordinated the Micronutrients Measurement Quality Assurance Program (MMQAP) for laboratories that measure fat- and water-soluble vitamins and carotenoids in human serum and plasma. The MMQAP provides participants with measurement comparability assessment through use of interlaboratory studies, Standard Reference Materials (SRMs) and control materials, and methods development and validation. Serum-based samples with assigned values for the target analytes (retinol, alphatocopherol, gamma/beta-tocopherol, trans- and total beta-carotene, and ascorbic acid) and performance-evaluation standards are distributed by NIST to laboratories for analysis.

Participants use the methodology of their choice to determine analyte content in the control and study materials. Participants provide their data to NIST, where it is compiled and evaluated for trueness relative to the NIST value, within-laboratory precision, and concordance within the participant community. NIST provides the participants with a technical summary report concerning their performance for each exercise and suggestions for methods development and refinement. Participants who have concerns regarding their laboratory's performance are encouraged to consult with the MMQAP coordinators.

All MMQAP interlaboratory studies consist of individual units of batch-prepared samples that are distributed to each participant. For historical reasons these studies are referred to as "Round Robins". The MMQAP program and the nature of its studies are described elsewhere. [1]

\section{Round Robin XVIII: Fat-Soluble Vitamins and Carotenoids in Human Serum}

Participants in the MMQAP Fat-Soluble Vitamins and Carotenoids in Human Serum Round Robin XVIII comparability study (hereafter referred to as RR18) received three lyophilized human serum test samples for analysis. Unless multiple vials were previously requested, participants received one vial of each serum. These sera were shipped on dry ice to participants in January 1990. The communication materials included in the sample shipment are described in Appendix A.

Participants are requested to report values for all fat-soluble vitamin-related analytes that are of interest to their organizations. Not all participants report values for the target analytes, and some participants report values for non-target analytes.

The final report delivered to every participant in RR18 is reproduced as Appendix B. This report included:

- Our analysis of the participants' results.

- Tabular presentations of all participant results.

- Graphical presentations of interlaboratory precision over time. These Figures are no longer available.

Each participant also received an "Individualized Report" that graphed their results for selected analytes. These figures are no longer available.

Appendix C lists all of the measurement results reported for RR18 in a more accessible format. 


\section{Round Robin XIX: Fat-Soluble Vitamins and Carotenoids in Human Serum}

Participants in the MMQAP Fat-Soluble Vitamins and Carotenoids in Human Serum Round Robin XIX comparability study (hereafter referred to as RR19) received three lyophilized human serum test samples for analysis. Unless multiple vials were previously requested, participants received one vial of each material. These sample materials were shipped on dry ice to participants in May 1990. The communication materials included in the sample shipment are described in Appendix D.

Participants are requested to report values for all fat-soluble vitamin-related analytes that are of interest to their organizations. Not all participants report values for the target analytes, and some participants report values for non-target analytes.

The final report delivered to every participant in RR19 is reproduced as Appendix E. This report included:

- Our analysis of the participants' results.

- Tabular presentations of all participant results.

- Graphical presentations of interlaboratory precision over time.

Each participant also received an "Individualized Report" that graphed their results for selected analytes. These figures are no longer available.

Appendix F lists all of the measurement results reported for RR19 in a more accessible format.

\section{Round Robin XX: Fat-Soluble Vitamins and Carotenoids in Human Serum}

Participants in the MMQAP Fat-Soluble Vitamins and Carotenoids in Human Serum Round Robin XX comparability study (hereafter referred to as RR20) received two liquid-frozen and three lyophilized human sera. Unless multiple vials were previously requested, participants received one vial of each material. These sample materials were shipped on dry ice to participants in August 1990. The communication materials included in the sample shipment are described in Appendix G.

Participants are requested to report values for all fat-soluble vitamin-related analytes that are of interest to their organizations. Not all participants report values for the target analytes, and some participants report values for non-target analytes.

It is probable that a preliminary report for RR20 was mailed to all participants shortly before the NIST/NCI Micronutrients Analysis Workshop held on November 15, 1990. However, no version of either the letter or the preliminary report is available. Likewise, only the tabular parts of the Final Report for RR20 are available; these tables are reproduced as Appendix H.

It is probable that each participant also received an "Individualized Report" that graphically analyzes their results for selected analytes. These figures are no longer available.

Appendix I lists all of the measurement results reported for RR20 in a more accessible format. 


\section{References}

1 Duewer DL, Brown Thomas J, Kline MC, MacCrehan WA, Schaffer R, Sharpless KE, May WE, Crowell JA. NIST/NCI Micronutrients Measurement Quality Assurance Program: Measurement Repeatabilities and Reproducibilities for Fat-Soluble Vitamin-Related Compounds in Human Sera. Anal Chem 1997;69(7):1406-1413. 


\section{Appendix A. Shipping Package Inserts for RR18}

Two items were included in each package shipped to an RR18 participant:

- Cover letter. The original letter has been lost. It would have described the three lyophilized sample materials (sera 120 to 122), ethanolic solution (sample 123), and three ethanolic calibration solutions distributed for the study, given guidance on reconstituting the serum samples and use of the calibration solutions, stated that results were due March 5, 1990 and to whom they should be sent, and who to contact with technical questions.

While there is no record of the cover letter, page A2 reproduces a letter sent at the same time as the Final Report for Round Robin XVII (RR17) that describes the RR18 materials. This notification letter was sent to laboratories that had not reported results for RR17; similar descriptions were included in the RR17 Final Reports provided to laboratories that had participated in RR17. These letters were sent about two weeks before the packages were to be shipped.

- Datasheets. Pages A3 and A4 reproduce the form. The first page of the form is for reporting the primary-focus analytes: retinol, $\alpha$-tocopherol, and total and trans- $\beta$ carotene. The second page is for reporting three "optional" analytes: retinyl palmitate, $\gamma$-tocopherol, and lycopene. In the absence of other information, the lycopene results are assumed to report total lycopene.

These items were attached to the shipping box. 
January 4, 1990

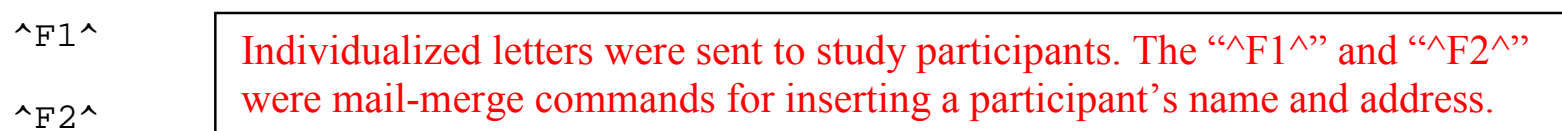

HAPPY NEW YEAR!

Samples for analysis of ROUND ROBIN XVIII will be shipped to you on January 16, 1990, and will include three serum samples and an ethanol solution of retinol, $\alpha$-tocopherol, and $\beta$-carotene. Analyze the serum samples as you normally do. We also invite you to provide us with data on retinol palmitate, lycopene, and $\gamma$-tocopherol in these samples.

Please complete your serum analyses before you analyze the unknown solution. For analysis of the unknown solution, we ask that you calibrate your system with the set of solution standards provided. Quantitation should be accomplished by comparing the response from the unknown with that from the calibration solutions. No extractions, additions of internal standards, or solvent exchanges should be required for labs using reversed-phase systems. The assigned values for the target analytes in calibration solutions to be used for analysis of the unknown solution follows:

\section{$(\mu \mathrm{g} / \mathrm{mL})$}

$\underline{\text { RETINOL }} \underline{\alpha \text {-TOCOPHEROL }} \quad \underline{\text { TRANS } \beta \text {-CAROTENE }}$

$\begin{array}{lccc}\text { Solution } 10 & 0.195 & 2.22 & 0.135 \\ \text { Solution } 11 & 0.977 & 11.1 & 0.945 \\ \text { Solution } 12 & 1.95 & 22.2 & 1.62\end{array}$

These solutions should be allowed to equilibrate at room temperature for at least 4 hours, but no more than 24 hours prior to use.

Results from the analysis of RR-XVIII are due by March 5, 1990.

Sincerely,

Willie E. May, Ph.D.

Chief

Organic Analytical Research Division

Center for Analytical Chemistry

Cc: Robert Schaffer

Winfred Malone 


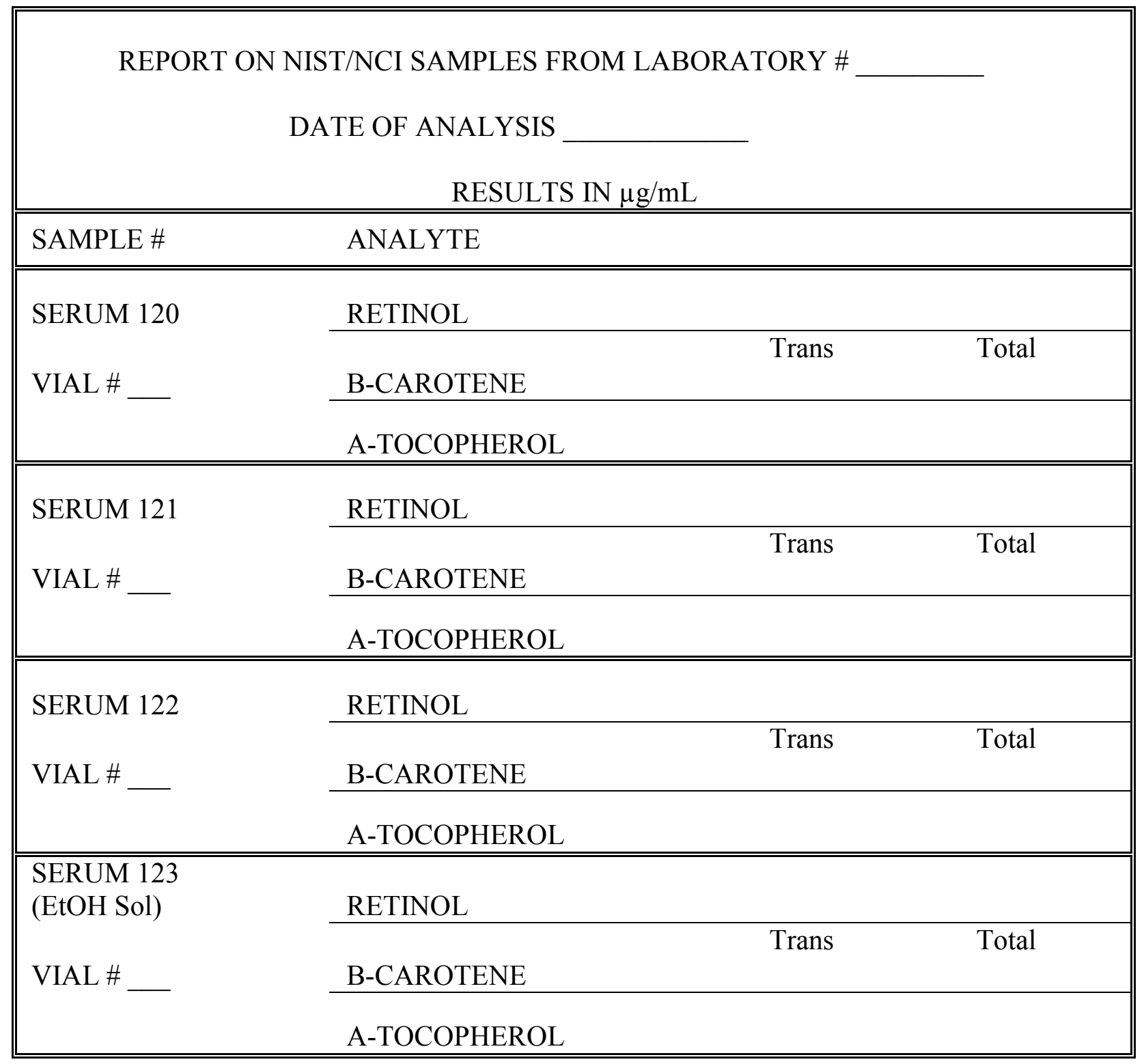

Add $1.0 \mathrm{~mL}$ of Distilled water to the Serum Samples. 


\begin{tabular}{|c|c|}
\hline \multicolumn{2}{|c|}{$\begin{array}{l}\text { REPORT ON NIST/NCI SAMPLES FROM LABORATORY \# } \\
\text { OPTIONAL ANALYTES } \\
\quad \text { DATE OF ANALYSIS }\end{array}$} \\
\hline \multicolumn{2}{|r|}{ RESULTS IN $\mu \mathrm{g} / \mathrm{mL}$} \\
\hline SAMPLE \# & ANALYTE \\
\hline \multirow{3}{*}{$\begin{array}{l}\text { SERUM } 120 \\
\text { VIAL \# }\end{array}$} & RETINYL PALMITATE \\
\hline & LYCOPENE \\
\hline & GAMMA-TOCOPHEROL \\
\hline \multirow{3}{*}{$\begin{array}{l}\text { SERUM } 121 \\
\text { VIAL \# }\end{array}$} & RETINYL PALMITATE \\
\hline & LYCOPENE \\
\hline & GAMMA-TOCOPHEROL \\
\hline \multirow{3}{*}{$\begin{array}{l}\text { SERUM } 122 \\
\text { VIAL \# }\end{array}$} & RETINYL PALMITATE \\
\hline & LYCOPENE \\
\hline & GAMMA-TOCOPHEROL \\
\hline
\end{tabular}




\section{Appendix B. Final Report for RR18}

The following six pages are the available components of the final report for RR18 as provided to all participants. The original report consisted of:

- A cover letter and discussion.

- Tables 1 to 3 that list results and various summary values for retinol, $\alpha$-tocopherol, and total $\beta$-carotene.

- Four unnumbered tables that list results and simple summary statistics for retinyl palmitate, lycopene, $\gamma$-tocopherol, and trans- $\beta$-carotene. Note: the cover letter states that the first three of these analytes constitute "Table 5"; the trans- $\beta$-carotene values were doubtless intended to be "Table 4."

- Graphical presentations of interlaboratory precision over time for retinol, $\alpha$-tocopherol, and total $\beta$-carotene.

When the isomeric form of an analyte is not specified, it is likely that most participants reported "total" (the sum of all isomers.) Since resolution of $\gamma$-tocopherol and $\beta$-tocopherol is challenging, the results reported as $\gamma$-tocopherol can be confidently assumed to be $\gamma / \beta$-tocopherol.

The graphical presentations for RR18 are no longer available; however, they would have been very similar to the graphs in the Final Report for RR19 in Appendix E.

Due to the complex formatting used in the Tables, the originally listed laboratory codes have been deleted without replacement. However, Appendix $\mathrm{C}$ provides a complete listing of the RR18 results where the original codes have been altered to ensure confidentiality. Appendix $\mathrm{C}$ also provides more relevant summary statistics. 
May 18, 1990

Dear Colleague:

Please pardon our delay in providing you with the results from Round-Robin XVIII. Tables 1-4 provide the usual compilation of individual laboratory results and a summary of the data for the determination of serum/plasma levels of retinol, $\alpha-$ tocopherol, total $\beta$-carotene, and trans $\beta$-carotene, respectively. Figures 1-3 provide an overall picture of the interlaboratory measurement capability (with respect to precision) for retinol, $\alpha$-tocopherol, and $\beta$-carotene over the past four years. Table 5 provides data compilations and summaries for serum/plasma determinations of three new analytes: retinyl palmitate, $\gamma$-tocopherol, and lycopene. As one might expect, interlaboratory variability is much greater (2-3 times) for the three new analytes than for our three core analytes.

As usual, the right side of Tables 1-3 provides data for use in evaluating your lab's performance in the study. The bias values given are based on the interlaboratory grand mean values for each analyte. The NIST value, based on measurements at NIST using one method only, is provided also for your information but not considered in computing the grand means. For retinol and $\alpha$-tocopherol, the relatively close agreement between the NIST and the grand mean values allows us to continue the convention of 0-5\% bias representing EXCEPTIONAL performance, 6-10\% ACCEPTABLE performance, 11-20\% MARGINAL performance, and $>20 \%$ UNACCEPTABLE with respect to the current state-of-the-practice for measurement of these analytes in serum/plasma.

Data reported for $\beta$-carotene is much less precise, and the biases between the grand mean and the NIST values are relatively large, $+19,-41$, and $-19 \%$ for serum samples 120,121 , and 122 respectively. The respective biases for retinol were 6,7 , and $7 \%$ and for $\alpha$-tocopherol $+3,+7$, and $-4 \%$. The reduced precision and apparent bias associated with the serum $\beta$-carotene data make individual laboratory performance difficult to evaluate. We hope that these data are anomalous and that $\beta$-carotene results from RR-XIX will be more in line with those from recent studies. If the imprecision continues and bias between the grand means and NIST values remains, round-robin studies and NIST laboratory studies will be designed to address the problem.

For the third time, results from the solution experiment perplex us. For all three analytes, the data from the analysis of the solution are more scattered than those for the three serum samples. For $\beta$-carotene this is understandable. First, after conversations with many of you concerning the non-linearity of the response from the three calibration solutions the we supplied to you, we observed that $B$-carotene does not completely redissolve in calibration solutions 11 and 12 by simply allowing them to remain at room temperature for 4 hours as you were instructed to do; instead 12 hours at room temperature or ultrasonic agitation for 10 minutes is necessary. Second, we observed $\beta$-carotene degradation products in several vials of the unknown solution that you were asked to analyze. From our analyses of 12 randomly selected vials, we obtained eight distinctly different values for ß-carotene, but identical values for retinol and $\alpha$-tocopherol. We found that the integrity and solubility of retinol and $\alpha$-tocopherol in both the calibration solutions and the unknown solutions to be good. In summary, scatter in the solution data for $\beta$-carotene is understandable due both to problems associated with dissolution of $\beta$-carotene in the calibration solutions provided 
to you and what appears to be degradation of $\beta$-carotene in the unknown solution. Reasons for the scatter observed in the solution data for retinol and $\alpha$-tocopherol are not apparent.

As you probably recall, the solution experiments were designed to serve two purposes: (1) to determine the relative contributions of solvent extraction/solvent exchange and chromatographic analysis to the overall 6-10\% CV for retinol and $\alpha$-tocopherol analyses and (2) to evaluate the need for you to modify your chromatographic systems in order to obtain values for individual $\beta$-carotenoid isomer determinations. Since the precision obtained from the determination of retinol and $\alpha$-tocopherol in ethanolic solutions in this and previous round-robins has curiously been worse than for serum analyses, it is certainly not possible to isolate the relative contributions of sample preparation and chromatographic analyses to the total variance. Therefore, until a better experiment can be designed to isolate these relative contributions, CV's of roughly 6-7\% appear to be the limit for interlaboratory serum determinations of retinol and $\alpha$-tocopherol. The integrity of both the calibration solutions and unknown sample preclude any substantive evaluation of $\beta$-carotene data from sample 123. Problems associated with total- and $\beta$-carotene isomer determination will continue to be a major focus in these studies.

Results are due back to NIST July 6.

Sincerely,

Willie E. May

Chief

Organic Analytical Research Division

Center for Analytical Chemistry 


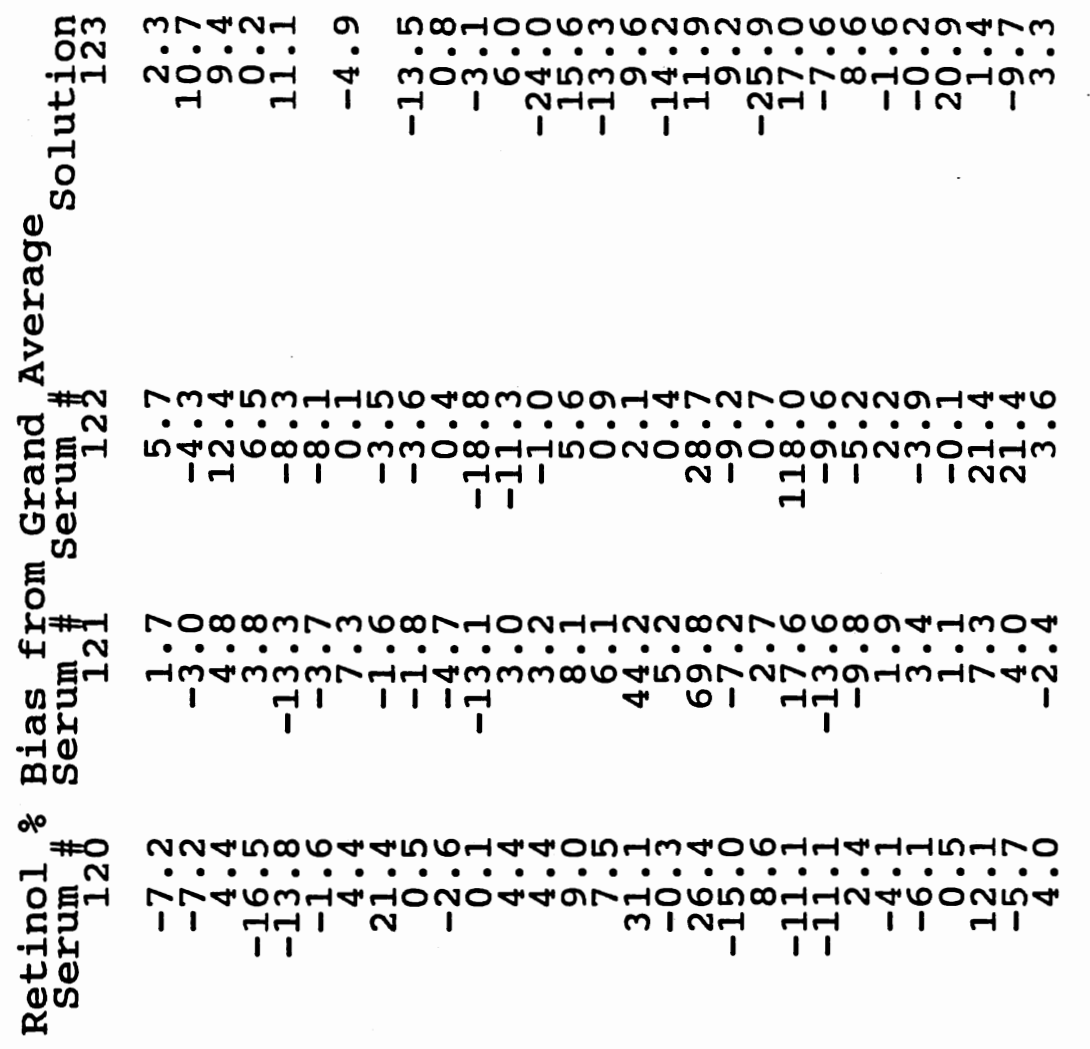
$\%$
⿷匚⿳丨コ丨

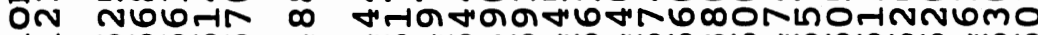

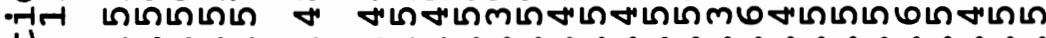 I ó000 $\dot{0} 0000000000000000000$ 깅

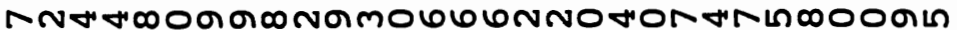

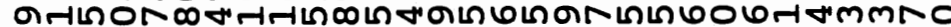

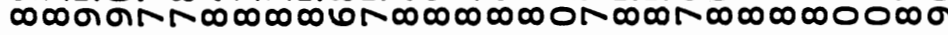
000000000000000-100-10000 *

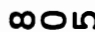
$+\infty$ $\infty 0 \sigma$ $\dot{0}$

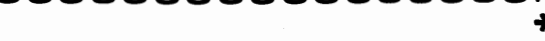
*

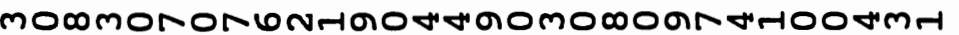

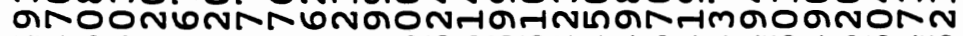

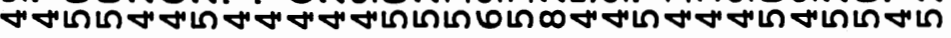

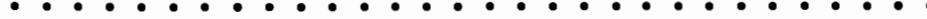
000000000000000000000000000000

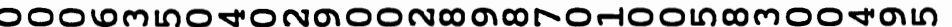

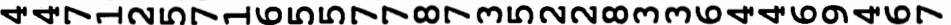

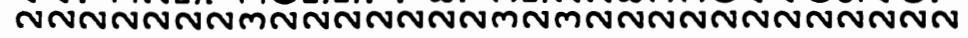
00000000000000000000000000 *

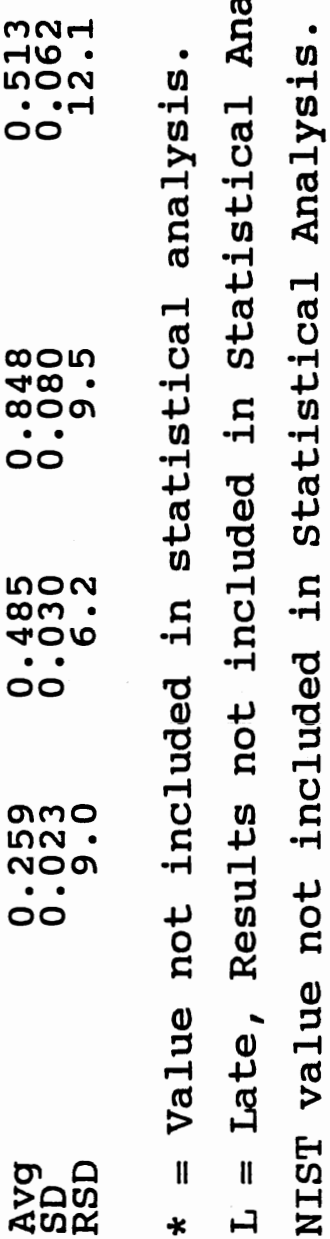


do

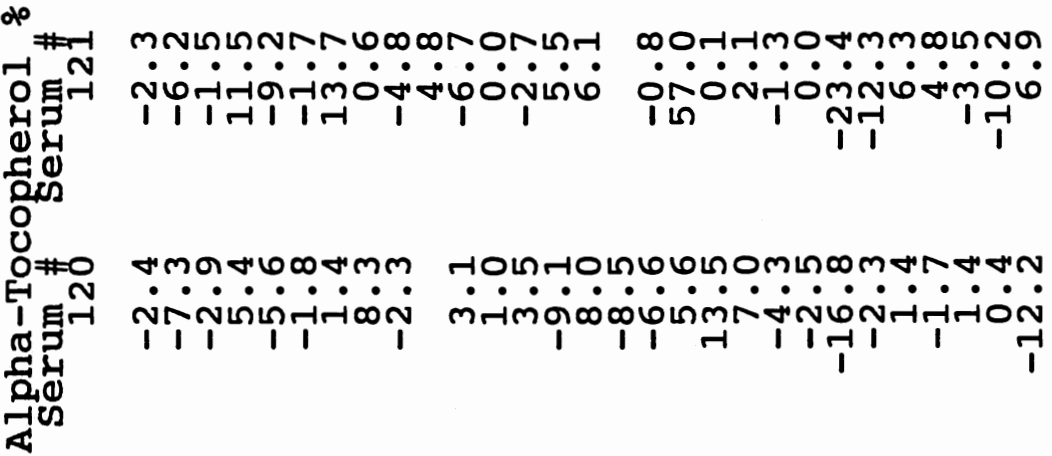

\#

NDNDO

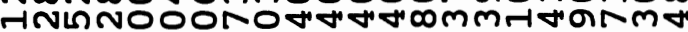

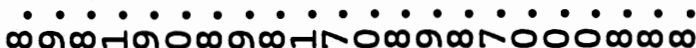

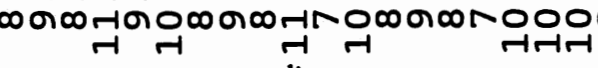

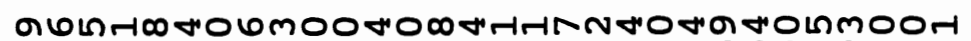

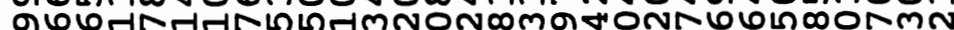

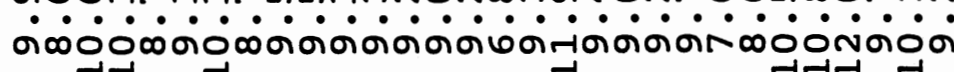

Nom $0 \infty$ $\cdot \infty \infty$ नत्म म1

$$
\text { * }
$$

mintaroneomporna

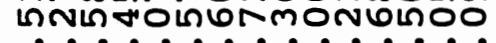
GOVNGONGONGGONA

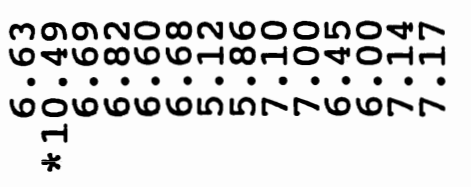

$$
\text { ต่อ }
$$$$
+
$$

HoghuाOMN A

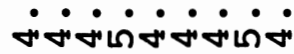

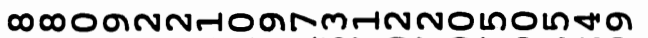

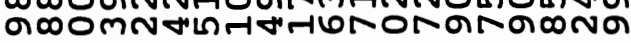

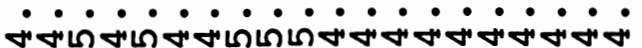
*

aro तN: बंन्म oio

$\infty \mathrm{m} n$ ம٪? மீ 10

mon $\infty$ N. $\rightarrow 0^{\circ}$

H 

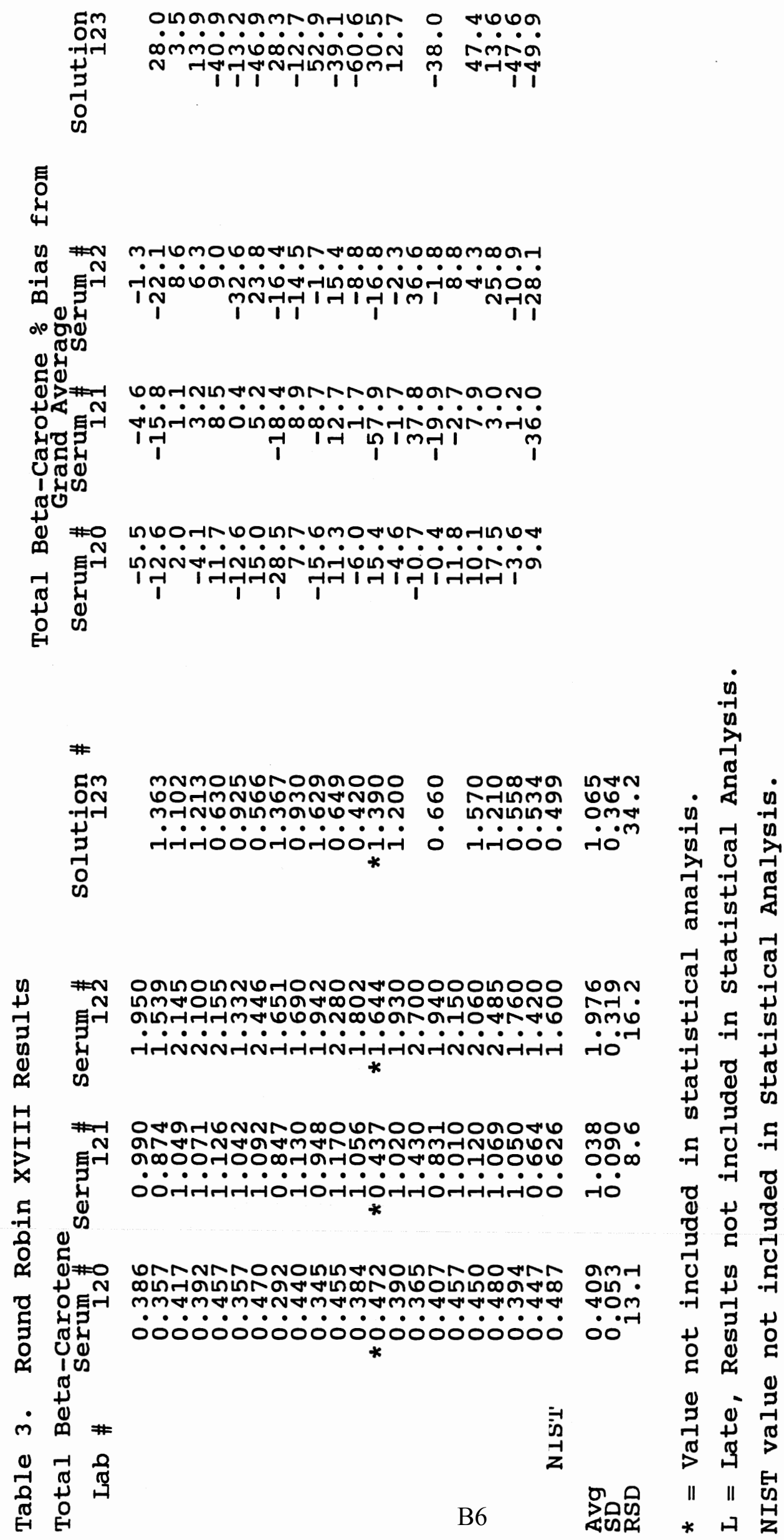

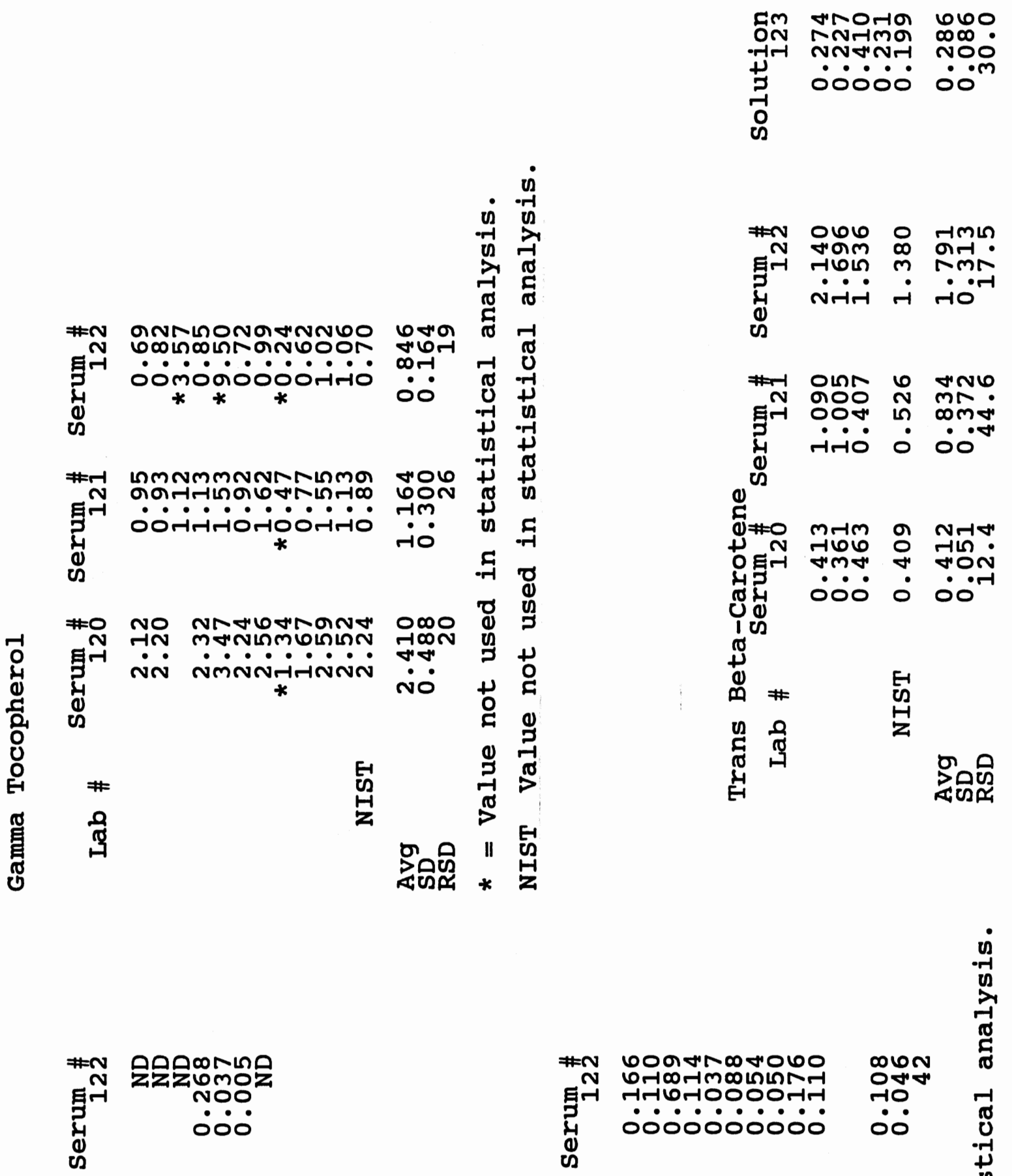

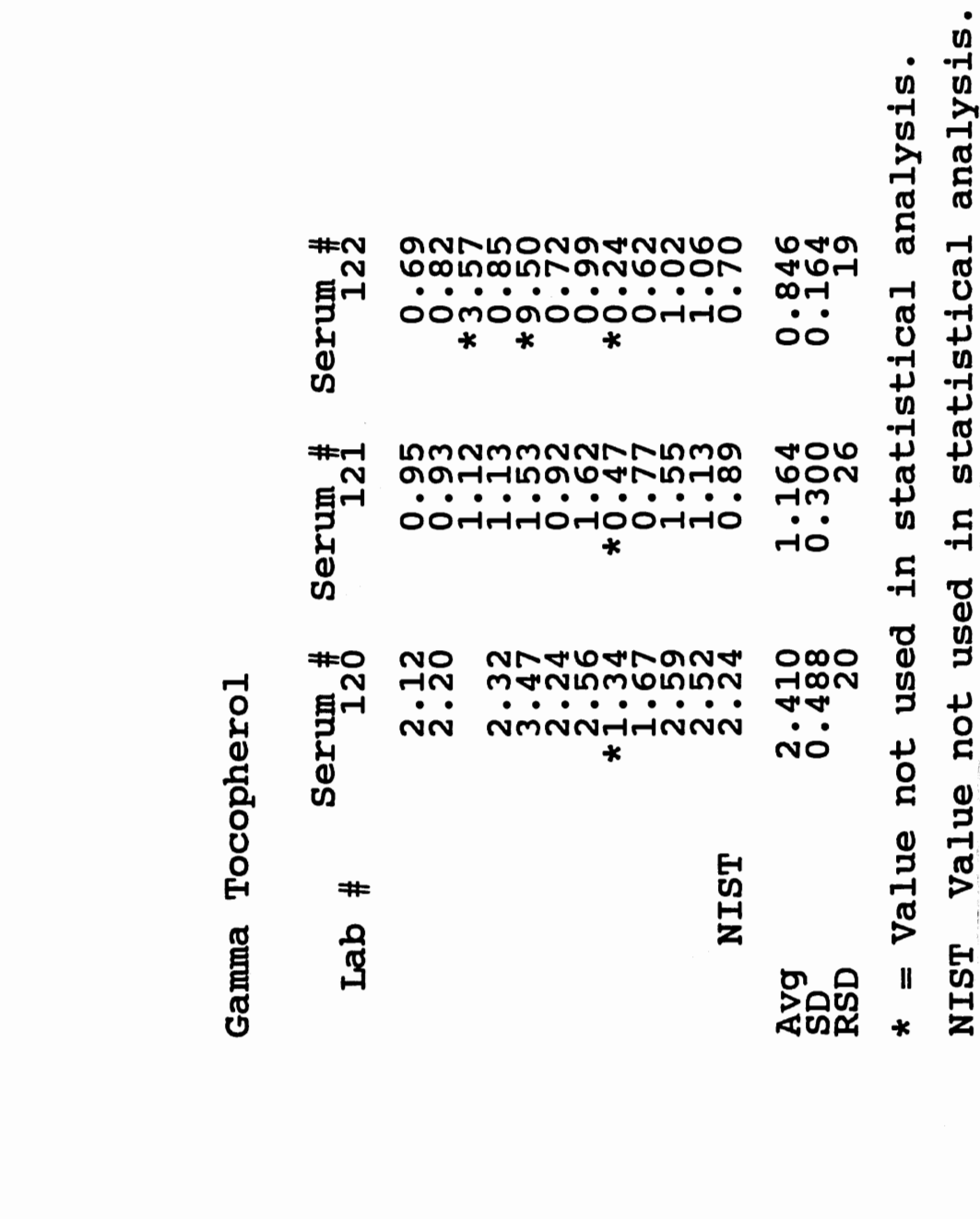
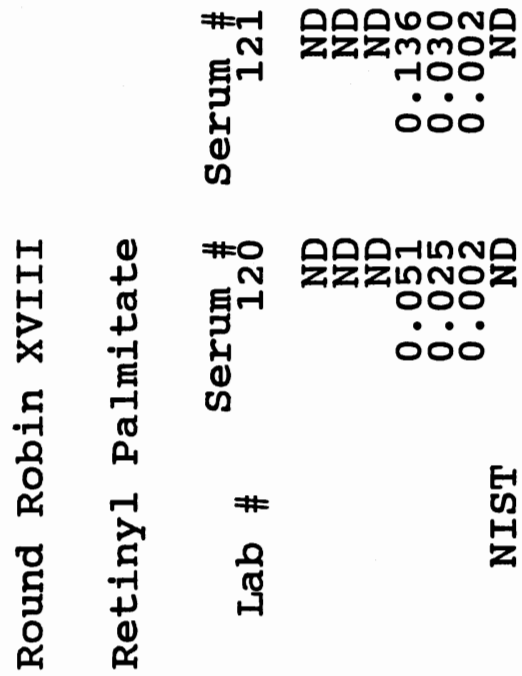

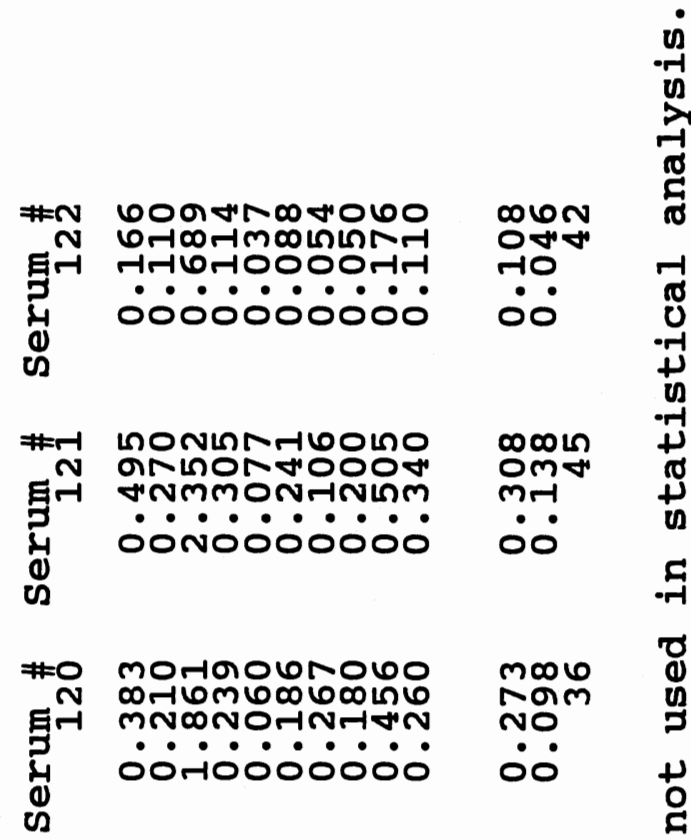
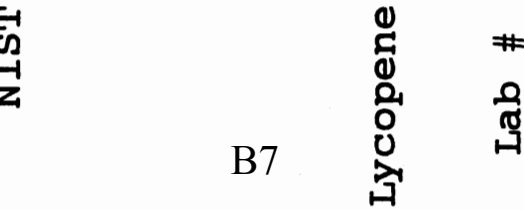


\section{Appendix C. Updated “All-Lab Report” for RR18}

The following four pages are a revised version of an "All-Lab" report for RR18. This report has three parts:

- pages 1 and 2 list results for all analytes reported at least twice, counting both participants and NIST analysts.

- $\quad$ page 3 provides a legend for pages 1 and 2 .

- page 4 summarizes each participants' performance for retinol, $\alpha$-tocopherol, and total $\beta$-carotene. These summaries are compatible with the percent bias evaluation advice given in the RR18 Report. However, the current bias summaries are estimated relative to the median of all reported values for each analyte in each serum rather than to the "Trimmed Average" used in the original and detailed in Appendix B. These original reference values were estimated from on-time results, with subjective exclusion of results deemed non-representative.

To ensure confidentiality, the laboratory identifiers used in this "All-Lab Report" have been altered from those used in RR18. The only attributed results are those reported by NIST. The NIST results are not used in the assessment of the consensus summary results of the study.

Note: The results designated NISTa in this updated All-Lab report are designated as NIST in the Tables of Appendix B. 


\section{Round Robin XVIII Laboratory Results}

\begin{tabular}{|c|c|c|c|c|c|c|c|c|c|c|c|c|c|c|c|c|}
\hline \multirow[b]{2}{*}{$\mathrm{Lab}$} & \multicolumn{4}{|c|}{ Total Retinol } & \multicolumn{4}{|c|}{ Retinyl Palmitate } & \multicolumn{4}{|c|}{$\alpha$-Tocopherol } & \multicolumn{4}{|c|}{ ү/ß-Tocopherol } \\
\hline & 120 & 121 & 122 & 123 & 120 & 121 & 122 & 123 & 120 & 121 & 122 & 123 & 120 & 121 & 122 & 123 \\
\hline FSV-BA & 0.278 & 0.514 & 0.856 & 0.445 & 0.025 & 0.030 & 0.037 & & 5.22 & 7.09 & 9.24 & 8.1 & & & & \\
\hline FSV-BD & 0.270 & 0.520 & 0.849 & 0.488 & & & & & 4.90 & 7.60 & 10.10 & 8.5 & & & & \\
\hline FSV-BE & 0.270 & 0.499 & 0.753 & 0.544 & & & & & 5.00 & 6.50 & 9.20 & 9.0 & & & & \\
\hline FSV-BF & 0.260 & 0.476 & 0.818 & 0.444 & & & & & 4.72 & 6.36 & 9.53 & 8.1 & 2.3 & 1.13 & 0.85 & \\
\hline FSV-BG & 0.270 & 0.508 & 0.954 & & & & & & 4.69 & 6.58 & 10.65 & 9.7 & & & & \\
\hline FSV-BH & 258 & 0.510 & 0.852 & 0.440 & $<0.02$ & $<0.02$ & $<0.02$ & & 4.51 & 6.63 & 9.31 & 8.0 & 2.2 & 0.95 & 0.72 & \\
\hline FSV-BI & 240 & 0.470 & 0.812 & 0.546 & nd & nd & nd & & 4.48 & 6.27 & 8.66 & 9.9 & 2.2 & 0.93 & 0.82 & \\
\hline FSV-BJ & 243 & 0.501 & 0.815 & 0.512 & & & & & 4.75 & 7.00 & 10.85 & 10.4 & 2.5 & 1.13 & 1.06 & \\
\hline FSV-BL & 267 & 0.482 & 0.798 & 0.505 & & & & & 4.96 & 6.38 & 8.71 & 9.1 & & & & \\
\hline FSV-BM & 248 & 0.494 & 0.867 & 0.505 & & & & & 4.90 & 7.10 & 10.50 & 10.1 & & & & \\
\hline FSV-BY & 0.240 & 0.493 & 0.897 & 0.525 & $n d$ & $n d$ & $n d$ & & 4.71 & 6.53 & 9.99 & 8.0 & 2.1 & 0.95 & 0.69 & \\
\hline FSV-BZ & & & & & & & & & 5.50 & 6.69 & 9.42 & 7.4 & & & & \\
\hline FSV-CA & 0.223 & 0.420 & 0.778 & 0.570 & & & & & 4.56 & 6.07 & 8.78 & 11.3 & & & & \\
\hline FSV-CJ & 216 & 0.503 & 0.904 & 0.514 & & & & & 5.09 & 7.45 & 10.11 & 8.7 & & & & \\
\hline FSV-CK & 327 & 0.823 & 1.092 & 0.574 & & & & & 5.10 & 10.49 & 11.97 & 11.4 & 2.6 & 1.62 & 0.99 & \\
\hline FSV-CM & & & & 0.480 & & & & & 4.71 & 6.68 & 9.74 & 9.8 & & & & \\
\hline FSV-CN & 314 & 0.477 & 0.819 & & & & & & 5.23 & 6.72 & 8.76 & & & & & \\
\hline FSV-CO & 255 & 0.467 & 0.780 & & $n d$ & $n d$ & nd & & 4.75 & 6.57 & 9.14 & & & & & \\
\hline FSV-CQ & 0.252 & 0.462 & 0.852 & 0.517 & 0.051 & 0.136 & 0.268 & & na & 7.00 & 9.50 & 9.3 & 3.5 & 1.53 & 9.50 & \\
\hline FSV-CV & 0.260 & 0.490 & 0.848 & 0.620 & & & & & 4.90 & 6.45 & 12.03 & 10.9 & & & & \\
\hline FSV-CY & 0.220 & 0.450 & 0.770 & 0.560 & & & & & 5.17 & 6.82 & 9.04 & 10.4 & 1.3 & 0.47 & 0.24 & \\
\hline FSV-DC & 0.282 & 0.544 & 0.896 & 0.593 & & & & & & & & & & & & \\
\hline FSV-DE & & & & & & & & & 4.88 & 6.68 & 9.34 & 9.5 & 2.2 & 0.92 & 0.72 & nd \\
\hline FSV-DG & 0.270 & 0.500 & 0.840 & 0.390 & & & & & 4.39 & 7.05 & 9.08 & 10.0 & & & & \\
\hline FSV-DH & 0.230 & 0.419 & 0.767 & 0.474 & 0.058 & 0.108 & 0.226 & & 4.02 & 5.12 & 7.69 & 8.4 & 2.0 & 0.73 & 0.74 & \\
\hline FSV-DL & 0.279 & 0.493 & 0.825 & 0.530 & & & & & & & & & & & & \\
\hline FSV-DT & 0.259 & 0.421 & 0.689 & 0.497 & & & & & 4.98 & 6.23 & 9.10 & 8.5 & & & & \\
\hline FSV-DY & 0.230 & 0.570 & 1.850 & 0.600 & & & & & & & & & & & & \\
\hline FSV-DZ & 0.339 & 0.699 & 0.866 & 0.562 & & & & & 4.42 & & 6.81 & 9.7 & & & & \\
\hline FSV-EB & 0.244 & 0.504 & 1.020 & 0.463 & & & & & 4.24 & 7.14 & 10.30 & 8.3 & & & & \\
\hline FSV-EF & 0.281 & 0.498 & 0.854 & 0.380 & & & & & 4.63 & 6.60 & 9.20 & 8.4 & 1.7 & 0.77 & 0.62 & \\
\hline FSV-EV & 0.290 & 0.520 & 1.030 & 0.520 & & & & & 4.85 & 6.00 & 9.70 & 8.7 & & & & \\
\hline FSV-FA & 0.265 & 0.437 & 0.804 & 0.557 & 0.002 & 0.002 & 0.005 & & 4.72 & 5.86 & 8.64 & 7.4 & 2.6 & 1.55 & 1.02 & \\
\hline$n$ & 30 & 30 & 30 & 28 & 4 & 4 & 4 & 0 & 29 & 29 & 30 & 28 & 12 & 2 & 12 & $c$ \\
\hline Min & 0.216 & 0.419 & 0.689 & 0.380 & .002 & 0.002 & 0.005 & & 4.02 & 5.12 & 6.81 & 7.39 & 1.34 & 0.47 & 0.24 & \\
\hline Mean & 0.263 & 0.505 & 0.885 & 0.513 & 0.034 & 0.069 & 0.134 & & 4.79 & 6.75 & 9.50 & 9.18 & 2.26 & 1. & 1.50 & \\
\hline Max & 0.339 & 0.823 & 1.850 & 0.620 & 0.058 & 0.136 & 0.268 & & 5.50 & 10.49 & 12.03 & 11.40 & 3.47 & 1.62 & 9.50 & \\
\hline SD & 0.029 & 0.079 & 0.201 & 0.059 & 0.026 & 0.063 & 0.132 & & 0.32 & 0.87 & 1.07 & 1.11 & 0.53 & 0.35 & 2.53 & \\
\hline $\mathrm{CV}$ & 11 & 16 & 23 & 12 & 75 & 92 & 99 & & 7 & 13 & 11 & 12 & 23 & 34 & 169 & \\
\hline $\begin{array}{l}\text { NISTa } \\
\text { NISTc }\end{array}$ & $\begin{array}{l}0.275 \\
0.244\end{array}$ & $\begin{array}{l}0.521 \\
0.433\end{array}$ & $\begin{array}{l}0.905 \\
0.726\end{array}$ & & $n d$ & $n d$ & $n d$ & & $\begin{array}{l}4.99 \\
4.47\end{array}$ & $\begin{array}{l}7.16 \\
6.18\end{array}$ & $\begin{array}{l}9.21 \\
8.27\end{array}$ & & $\begin{array}{l}2.2 \\
2.5\end{array}$ & $\begin{array}{l}0.89 \\
1.43\end{array}$ & $\begin{array}{l}0.70 \\
1.25\end{array}$ & \\
\hline Median & 0.260 & 0.496 & 0.849 & 0.516 & & & & & 4.75 & 6.63 & 3 & 9.05 & 2.22 & 95 & 0.78 & \\
\hline eSD & 0.026 & 0.029 & 0.068 & 0.062 & & & & & 0.31 & 0.53 & 0.82 & 1.22 & 0.39 & 0.27 & 0.18 & \\
\hline $\mathrm{eCV}$ & 10 & 6 & 8 & 12 & & & & & 7 & 8 & 9 & 13 & 18 & 28 & 24 & \\
\hline
\end{tabular}




\section{Round Robin XVIII Laboratory Results}

\begin{tabular}{|c|c|c|c|c|c|c|c|c|c|c|c|c|c|c|c|c|}
\hline \multirow[b]{2}{*}{ Lab } & \multicolumn{4}{|c|}{ Total $\beta$-Carotene } & \multicolumn{4}{|c|}{ trans- $\beta$-Carotene } & \multicolumn{4}{|c|}{ Total cis- $\beta$-Carotene } & \multicolumn{4}{|c|}{ Total Lycopene } \\
\hline & 120 & 121 & 122 & 123 & 120 & 121 & 122 & 123 & 120 & 121 & 122 & 123 & 120 & 121 & 122 & 123 \\
\hline FSV-BA & 0.455 & 1.17 & 2.28 & 0.65 & 0.413 & 1.090 & 2.140 & 0.274 & 0.042 & 0.080 & 0.140 & 0.375 & 0.19 & 0.24 & 0.088 & \\
\hline FSV-BD & & & & & & & & & & & & & & & & \\
\hline FSV-BE & & & & & & & & & & & & & & & & \\
\hline FSV-BF & 0.470 & 1.09 & 2.45 & 0.57 & & & & & & & & & 0.24 & 0.31 & 0.114 & \\
\hline FSV-BG & 0.417 & 1.05 & 2.15 & 1.10 & & & & & & & & & & & & \\
\hline FSV-BH & 0.384 & 1.06 & 1.80 & 0.42 & 0.361 & 1.005 & 1.696 & 0.227 & 0.023 & 0.051 & 0.106 & 0.193 & 0.39 & 0.55 & 0.154 & \\
\hline FSV-BI & 0.357 & 0.87 & 1.54 & 1.14 & & & & & & & & & 0.21 & 0.27 & 0.110 & \\
\hline FSV-BJ & 0.450 & 1.12 & 2.06 & 1.57 & & & & & & & & & 0.26 & 0.34 & 0.110 & \\
\hline FSV-BL & & & & & & & & & & & & & & & & \\
\hline FSV-BM & & & & & & & & & & & & & & & & \\
\hline FSV-BY & 0.386 & 0.99 & 1.95 & & & & & & & & & & 0.38 & 0.50 & 0.166 & \\
\hline FSV-BZ & & & & & & & & & & & & & & & & \\
\hline FSV-CA & & & & & & & & & & & & & & & & \\
\hline FSV-CJ & 0.392 & 1.07 & 2.10 & 1.21 & & & & & & & & & & & & \\
\hline FSV-CK & 0.472 & 0.44 & 1.64 & 1.39 & 0.463 & 0.407 & 1.536 & 0.410 & 0.009 & 0.029 & 0.109 & 0.980 & 0.27 & 0.11 & 0.054 & \\
\hline FSV-CM & & & & & & & & & & & & & & & & \\
\hline FSV-CN & 0.357 & 1.04 & 1.33 & 0.93 & & & & & & & & & & & & \\
\hline FSV-CO & 0.457 & 1.13 & 2.15 & 0.63 & & & & & & & & & & & & \\
\hline FSV-CQ & 0.292 & 0.85 & 1.65 & & & & & & & & & & 0.06 & 0.08 & 0.037 & \\
\hline FSV-CV & 0.480 & 1.07 & 2.49 & 1.21 & & & & & & & & & & & & \\
\hline FSV-CY & 0.390 & 1.02 & 1.93 & 1.20 & & & & & & & & & 0.18 & 0.20 & 0.050 & \\
\hline FSV-DC & 0.345 & 0.95 & 1.95 & 1.63 & & & & & & & & & & & & \\
\hline FSV-DE & & & & & & & & & & & & & & & & \\
\hline FSV-DG & 0.440 & 1.13 & 1.69 & 0.93 & & & & & & & & & & & & \\
\hline FSV-DH & 0.407 & 0.83 & 1.94 & 0.66 & & & & & & & & & 0.37 & 0.40 & 0.177 & \\
\hline FSV-DL & 0.451 & 1.50 & 1.49 & & & & & & & & & & & & & \\
\hline FSV-DT & & & & & & & & & & & & & & & & \\
\hline FSV-DY & 0.365 & 1.43 & 2.70 & & & & & & & & & & & & & \\
\hline FSV-DZ & & & & & & & & & & & & & & & & \\
\hline FSV-EB & 0.394 & 1.05 & 1.76 & 0.56 & & & & & & & & & & & & \\
\hline FSV-EF & & & & & & & & & & & & & & & & \\
\hline FSV-EV & & & & & & & & & & & & & & & & \\
\hline FSV-FA & 0.457 & 1.01 & 2.15 & & & & & 0.231 & & & & & 0.46 & 0.51 & 0.176 & \\
\hline $\mathrm{n}$ & 21 & 21 & 21 & 16 & 3 & 3 & 3 & 4 & 3 & 3 & 3 & 3 & 11 & 11 & 11 & 0 \\
\hline Min & 0.292 & 0.437 & 1.332 & 0.420 & 0.361 & 0.407 & 1.536 & 0.227 & 0.009 & 0.029 & 0.106 & 0.193 & 0.060 & 0.077 & 0.037 & \\
\hline Mean & 0.410 & 1.041 & 1.962 & 0.987 & 0.412 & 0.834 & 1.791 & 0.286 & 0.025 & 0.053 & 0.118 & 0.516 & 0.273 & 0.317 & 0.112 & \\
\hline Max & 0.480 & 1.495 & 2.700 & 1.629 & 0.463 & 1.090 & 2.140 & 0.410 & 0.042 & 0.080 & 0.140 & 0.980 & 0.456 & 0.548 & 0.177 & \\
\hline SD & 0.050 & 0.211 & 0.348 & 0.377 & 0.051 & 0.372 & 0.313 & 0.086 & 0.017 & 0.025 & 0.019 & 0.412 & 0.117 & 0.159 & 0.051 & \\
\hline $\mathrm{CV}$ & 12 & 20 & 18 & 38 & 12 & 45 & 17 & 30 & 69 & 48 & 16 & 80 & 43 & 50 & 46 & \\
\hline NISTa & 0.487 & 0.63 & 1.60 & & 0.409 & 0.526 & 1.383 & & 0.078 & 0.100 & 0.213 & & & & & \\
\hline NISTc & 0.448 & 0.98 & 1.71 & & 0.369 & 0.853 & 1.530 & & 0.080 & 0.124 & 0.175 & & 0.21 & 0.22 & 0.127 & \\
\hline Median & 0.407 & 1.050 & 1.950 & 1.016 & & & & & & & & & 0.260 & 0.305 & 0.110 & \\
\hline eSD & 0.065 & 0.104 & 0.303 & 0.536 & & & & & & & & & 0.119 & 0.156 & 0.083 & \\
\hline eCV & 16 & 10 & 16 & 53 & & & & & & & & & 46 & 51 & 75 & \\
\hline
\end{tabular}




\section{Round Robin XVIII Laboratory Results}

\section{Table Legend}

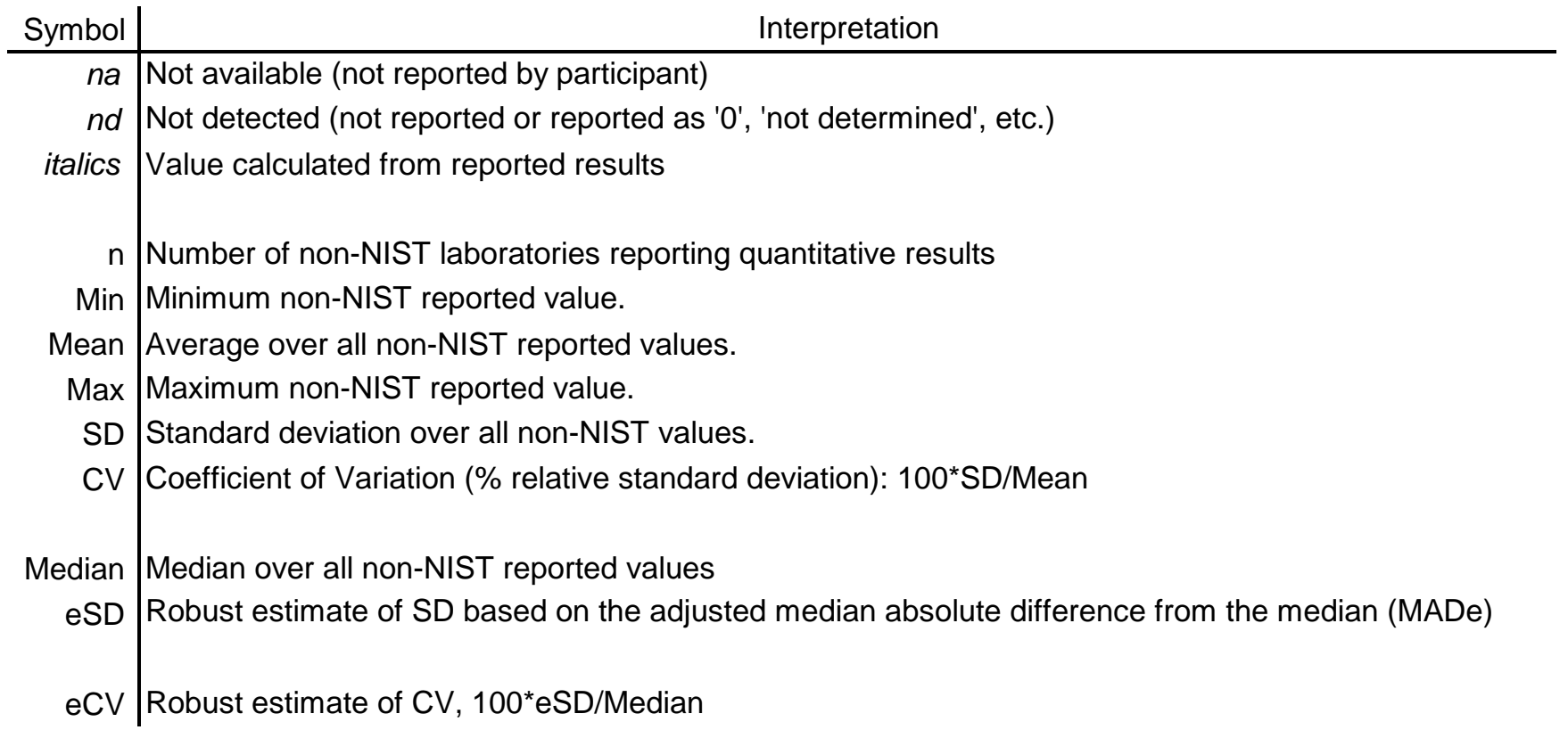




\section{Round Robin XVIII Laboratory Results}

\begin{tabular}{l|rrr|}
\cline { 2 - 4 } Lab & \multicolumn{1}{c}{ TR } & \multicolumn{1}{c}{$\mathrm{aT}$} & \multicolumn{1}{c|}{$\mathrm{bC}$} \\
\hline FSV-BA & $4 \pm 3$ & $5 \pm 6$ & $13 \pm 3$ \\
FSV-BD & $3 \pm 3$ & $9 \pm 6$ & \\
FSV-BE & $-2 \pm 8$ & $1 \pm 4$ & \\
FSV-BF & $-3 \pm 2$ & $-1 \pm 3$ & $15 \pm 11$ \\
FSV-BG & $6 \pm 5$ & $4 \pm 9$ & $4 \pm 5$ \\
FSV-BH & $1 \pm 2$ & $-2 \pm 3$ & $-4 \pm 4$ \\
FSV-BI & $-6 \pm 2$ & $-6 \pm 1$ & $-17 \pm 4$ \\
FSV-BJ & $-3 \pm 4$ & $7 \pm 8$ & $8 \pm 3$ \\
FSV-BL & $-2 \pm 4$ & $-2 \pm 6$ & \\
FSV-BM & $-1 \pm 3$ & $8 \pm 5$ & \\
FSV-BY & $-1 \pm 7$ & $2 \pm 5$ & $-4 \pm 3$ \\
FSV-BZ & & $6 \pm 9$ & \\
FSV-CA & $-13 \pm 4$ & $-6 \pm 2$ & \\
FSV-CJ & $-3 \pm 12$ & $9 \pm 3$ & $2 \pm 6$ \\
FSV-CK & $40 \pm 22$ & $31 \pm 26$ & $-19 \pm 37$ \\
FSV-CM & & $1 \pm 3$ & \\
FSV-CN & $4 \pm 14$ & $2 \pm 8$ & $-15 \pm 16$ \\
FSV-CO & $-5 \pm 3$ & $-1 \pm 1$ & $10 \pm 3$ \\
FSV-CQ & $-3 \pm 4$ & $4 \pm 3$ & $-21 \pm 7$ \\
FSV-CV & $0 \pm 1$ & $10 \pm 17$ & $16 \pm 13$ \\
FSV-CY & $-11 \pm 4$ & $3 \pm 6$ & $-3 \pm 2$ \\
FSV-DC & $8 \pm 2$ & & $-8 \pm 8$ \\
FSV-DE & & $1 \pm 1$ & \\
FSV-DG & $1 \pm 2$ & $-1 \pm 7$ & $1 \pm 12$ \\
FSV-DH & $-12 \pm 3$ & $-19 \pm 4$ & $-7 \pm 12$ \\
FSV-DL & $1 \pm 5$ & & $10 \pm 33$ \\
FSV-DT & $-11 \pm 10$ & $-1 \pm 6$ & \\
FSV-DY & $40 \pm 68$ & & $21 \pm 28$ \\
FSV-DZ & $24 \pm 20$ & $-17 \pm 14$ & \\
FSV-EB & $5 \pm 14$ & $2 \pm 12$ & $-4 \pm 5$ \\
FSV-EF & $3 \pm 4$ & $-1 \pm 1$ & \\
FSV-EV & $13 \pm 8$ & $-1 \pm 7$ & \\
FSV-FA & $-5 \pm 7$ & $-7 \pm 6$ & $6 \pm 9$ \\
\cline { 2 - 4 } NISTa & $6 \pm 1$ & $4 \pm 5$ & $-13 \pm 30$ \\
NISTC & $-11 \pm 4$ & $-8 \pm 3$ & $-3 \pm 12$ \\
\hline & & &
\end{tabular}

\begin{tabular}{r|l} 
Label & Definition \\
\hline Lab & Participant code \\
TR & Total Retinol \\
aT & a-Tocopherol \\
bC & Total b-Carotene \\
$\%$ Bias & (Mean \pm SD) of individual serum biases \\
Mean & Average of $\left(\mathrm{x}_{\mathrm{i}}-\right.$ Median $\left._{\mathrm{i}}\right) /$ Median $_{\mathrm{i}}$ \\
$\mathrm{SD}$ & Standard deviation of $\left(\mathrm{x}_{\mathrm{i}}\right.$-Median $\left._{\mathrm{i}}\right) /$ Median $_{\mathrm{i}}$ \\
$\mathrm{x}_{\mathrm{i}}$ & Result for analyte in serum \\
Median $_{\mathrm{i}}$ & Median of non-NIST results in serum \\
\end{tabular}

The original analysis listed \% Bias for each result for each serum calculated relative to the "Trimmed Core Lab Average" of that analyte in the serum. The summary values reported here are the (arithmetic mean \pm standard deviation) of each laboratory's reported results for the analyte estimated relative to each serum's median value.

The results for the calibration solution 123 do not necessarily reflect a participant's measurement performance for serum samples. Therefore, these summary values are calculated using only results for the four serum samples: 120, 121, and 122. 


\section{Appendix D. Shipping Package Inserts for RR19}

Two items were included in each package shipped to an RR19 participant:

- Cover letter. The original letter has been lost. It would have described the four lyophilized sample materials (sera 130 to 133) distributed for the study, given guidance on reconstituting these samples, stated that results were due July 6, 1990 and to whom they should be sent, and who to contact with technical questions.

- Datasheets. The following two pages reproduce the form. The first page of the form is for reporting the primary-focus analytes: retinol, $\alpha$-tocopherol, and total and trans- $\beta-$ carotene. The second page is for reporting three "optional" analytes: retinyl palmitate, $\gamma$-tocopherol, and lycopene. In the absence of other information, the lycopene results are assumed to report total lycopene.

These items were attached to the shipping box. 


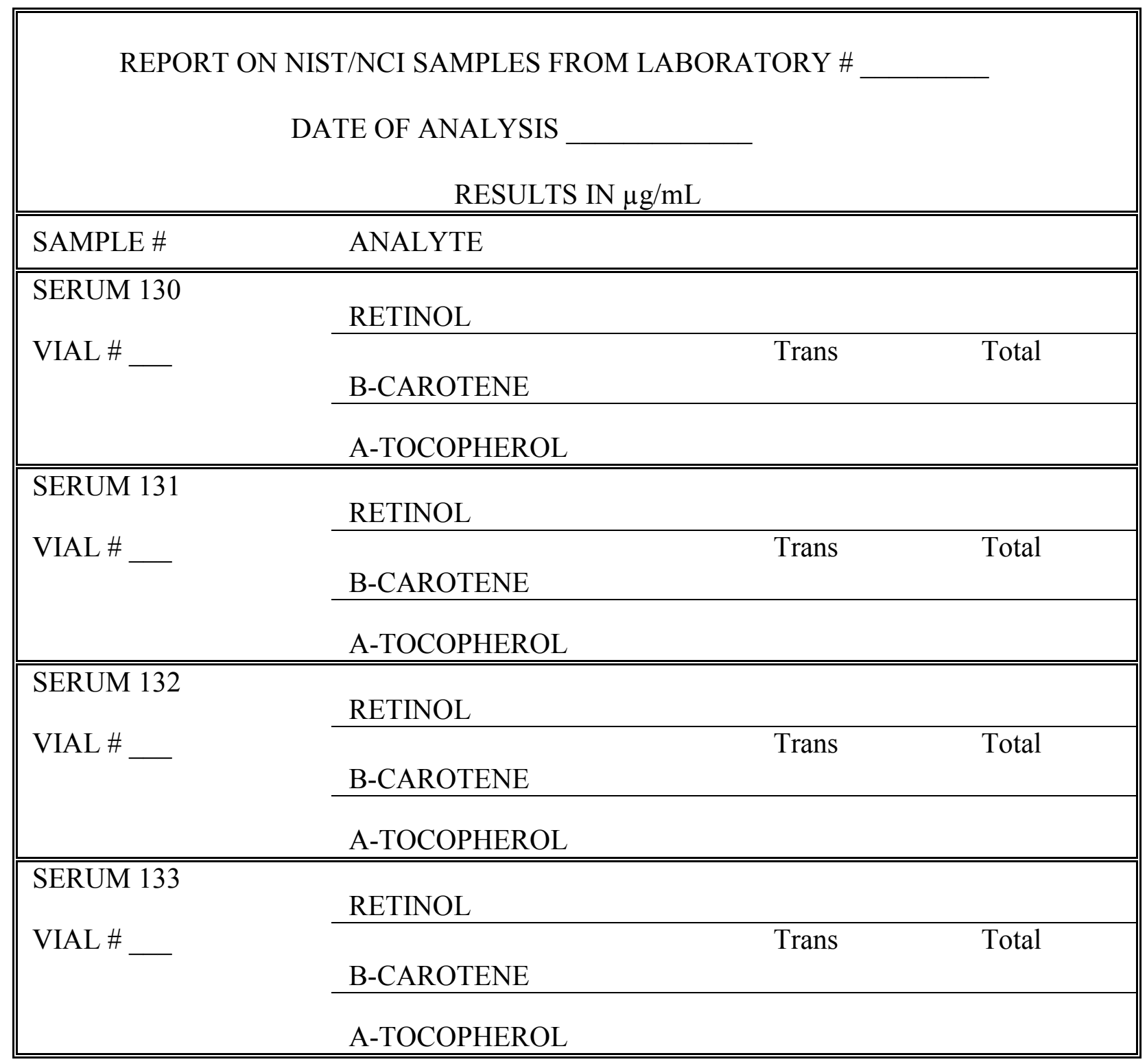

Add $1.0 \mathrm{~mL}$ of Distilled water to the Serum Samples. 


\begin{tabular}{|c|c|}
\hline \multicolumn{2}{|c|}{$\begin{array}{l}\text { REPORT ON NIST/NCI SAMPLES FROM LABORATORY \# } \\
\text { OPTIONAL ANALYTES } \\
\quad \text { DATE OF ANALYSIS }\end{array}$} \\
\hline \multicolumn{2}{|r|}{ RESULTS IN $\mu \mathrm{g} / \mathrm{mL}$} \\
\hline SAMPLE \# & ANALYTE \\
\hline \multirow{3}{*}{$\begin{array}{l}\text { SERUM } 130 \\
\text { VIAL \# }\end{array}$} & RETINYL PALMITATE \\
\hline & LYCOPENE \\
\hline & GAMMA-TOCOPHEROL \\
\hline \multirow{3}{*}{$\begin{array}{l}\text { SERUM } 131 \\
\text { VIAL \# }\end{array}$} & RETINYL PALMITATE \\
\hline & LYCOPENE \\
\hline & GAMMA-TOCOPHEROL \\
\hline \multirow{3}{*}{$\begin{array}{l}\text { SERUM } 132 \\
\text { VIAL \# }\end{array}$} & RETINYL PALMITATE \\
\hline & LYCOPENE \\
\hline & GAMMA-TOCOPHEROL \\
\hline \multirow{3}{*}{$\begin{array}{l}\text { SERUM } 133 \\
\text { VIAL \# }\end{array}$} & RETINYL PALMITATE \\
\hline & LYCOPENE \\
\hline & GAMMA-TOCOPHEROL \\
\hline
\end{tabular}




\section{Appendix E. Final Report for RR19}

The following eight pages are the final report for RR19 as provided to all participants:

- A cover letter and discussion.

- Tables 1 to 3 that list the results and various summary values for retinol, $\alpha$-tocopherol, and total $\beta$-carotene.

- Table 4 that lists the results and simple summary statistics for trans- $\beta$-carotene, retinyl palmitate, lycopene, and $\gamma$-tocopherol.

- Figures 1 to 3 that present interlaboratory precision over time for retinol, $\alpha$-tocopherol, and total $\beta$-carotene.

It is likely that most participants reported "total lycopene" (the sum of all isomers.) Since resolution of $\gamma$-tocopherol and $\beta$-tocopherol is challenging, the results reported as $\gamma$-tocopherol can be confidently assumed to be $\gamma / \beta$-tocopherol.

Due to the complex formatting used in the Tables, the originally listed laboratory codes have been deleted without replacement. However, Appendix F provides a complete listing of the RR19 results where the original codes have been altered to ensure confidentiality. Appendix F also provides more relevant summary statistics. 
Personalized letters were sent to participants.

This block contained their formal name and address.

\section{Dear (Personal name),}

By now you should have received a letter from Bob Schaffer regarding this years QA Workshop. I hope to see you here on November $15^{\text {th }}$. Discussions regarding our analysis of data from Round-Robin XIX follows.

Tables 1-3 each provide the usual compilation of both individual laboratory results and a summary of the interlaboratory data from the determination of serum/plasma levels of retinol, $\alpha$-tocopherol, total $\beta$-carotene, respectively. Table 4 provides data compilations and summaries for serum/plasma determinations of trans $\beta$-carotene and three new analytes: retinyl palmitate, $\gamma$-tocopherol, and lycopene. Upon inspection of the data reported for these analytes, you will readily notice that, as in Round-Robin XVIII, interlaboratory variability is much greater for the three new compounds than for our three core analytes for which QA/QC procedure and practices have been put in place. Variations in interlaboratory precision for the determination of serum/plasma levels of retinol, $\alpha$-tocopherol, and $\beta$-carotene respectively, over the past four years are shown in Figures 1-3. You are also provided with your lab's "Blind Control Charts" for each of the three core analytes.

As usual, the right side of Tables 1-3 provides data for use in evaluating your lab's performance in the study. The bias values given are based on the interlaboratory grand means for each analyte. NIST values are also provided for your information, but are not used in computing the grand means against which laboratory performance is being evaluated. A copy of a report describing NIST Analyses of Round Robin XIX samples is provided for your information. For all three analytes, the relatively close agreement between the NIST and grand mean values allows us to continue the convention of 0-5\% bias from the grand mean representing EXCEPTIONAL performance, 6-10\% ACCEPTABLE performance, 11-20\% MARGINAL performance, and $>20 \%$ UNACCEPTABLE with respect to the current state-of-the-practice for measurement of these analytes in serum/plasma.

Round-Robin XX samples will be shipped to you on September $10^{\text {th }}$. Results are required by October $22^{\text {nd }}$.

Sincerely,

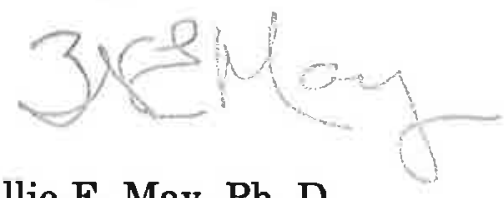

Willie E. May, Ph. D.

Chief

Organic Analytical Research Division

Center for Analytical Chemistry

Enclosures 
\#

目我

H ช

$N$

园

H

(5)

\#

E्त

S月-1

क

\#

옹

田

1)

4

$\#$

르

돈

En

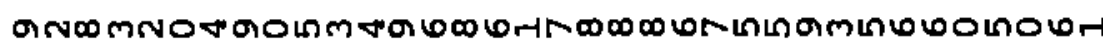

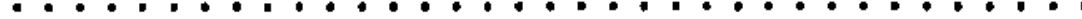
Iन

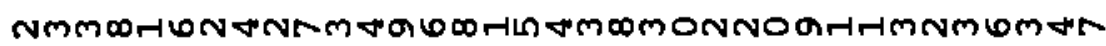
- :

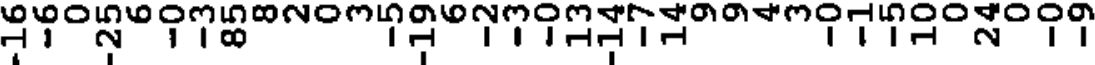

\$NWNHWH

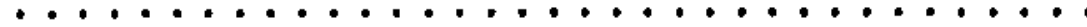

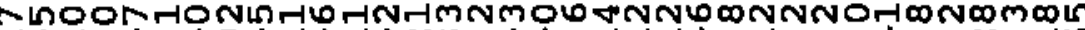

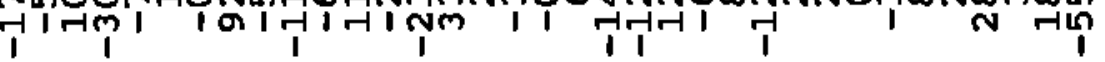

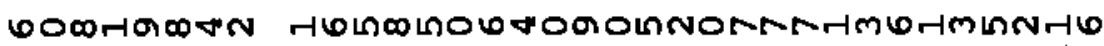

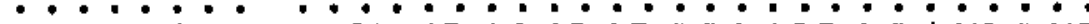

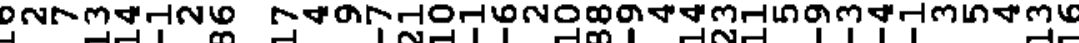
I

Lก ONN Zn+

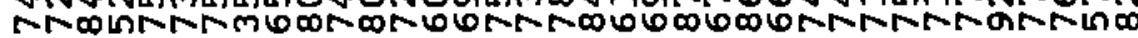

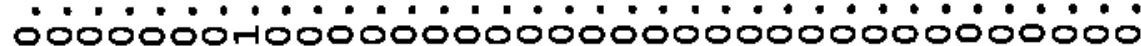

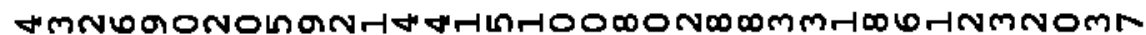
Hin-

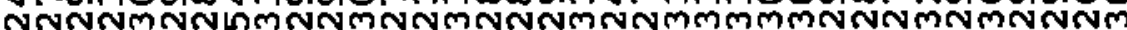

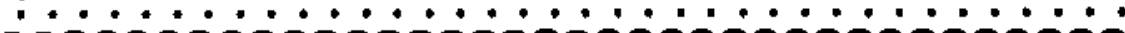
000000000000000000000000000000000000

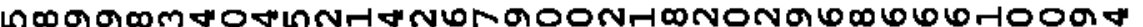
No

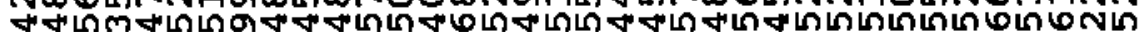
* +ก!

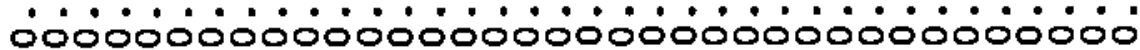

(⿻)

momonnto

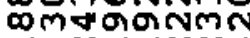
HNNHANA . . . $\cdot$.

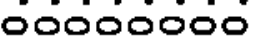

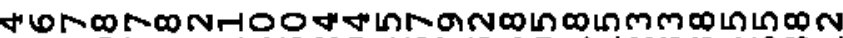
OMdor

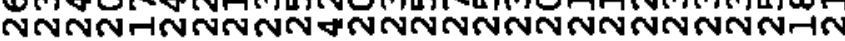

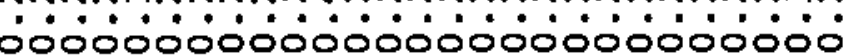

$\infty \mathrm{n}$ $m \infty \cdot$ $\mathrm{MOH}$ 00 (1)

$-\infty \infty$ arv. Non 00<smiles>CCC</smiles>

LNA -16. Inom ió

omन NN : NoO i० 
\# 岁

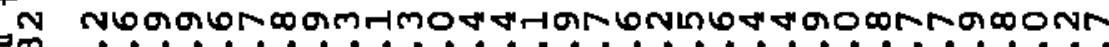

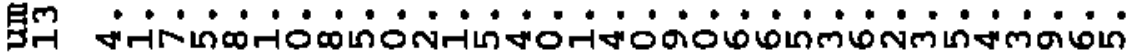

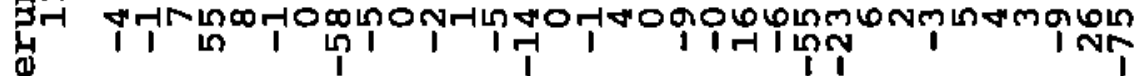

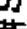

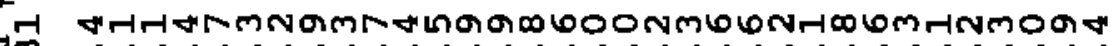

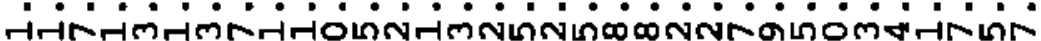

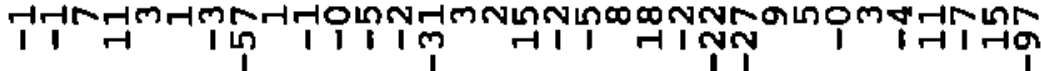

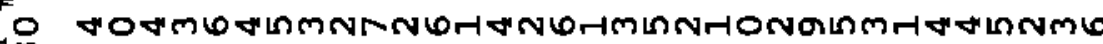

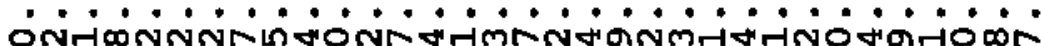

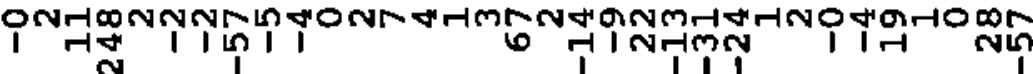
Tw w

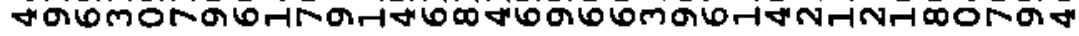

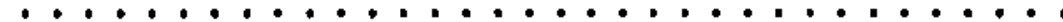

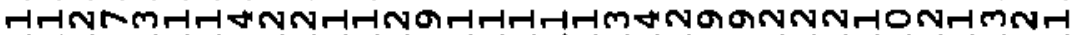

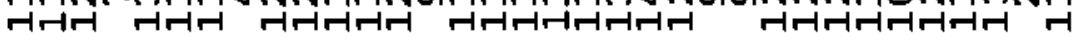

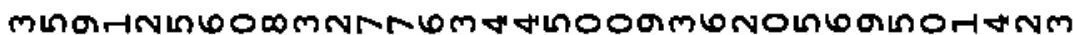

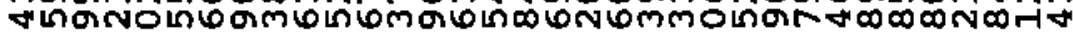

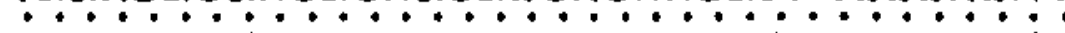

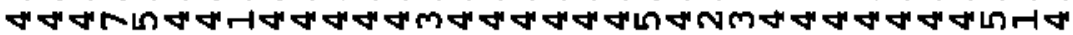

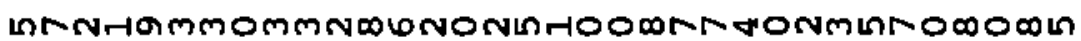

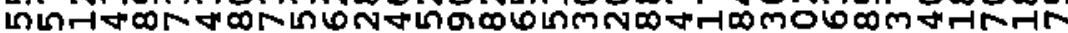$$
\text { lantovió }
$$

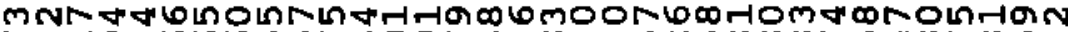

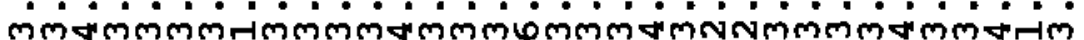
म $\infty$ $\infty$ N. - 0 न्नान

$\mathrm{Nmm}$ ט大? - $\rightarrow$ $+0$

$$
\text { \% }
$$

$106 \pi$ or. 60न

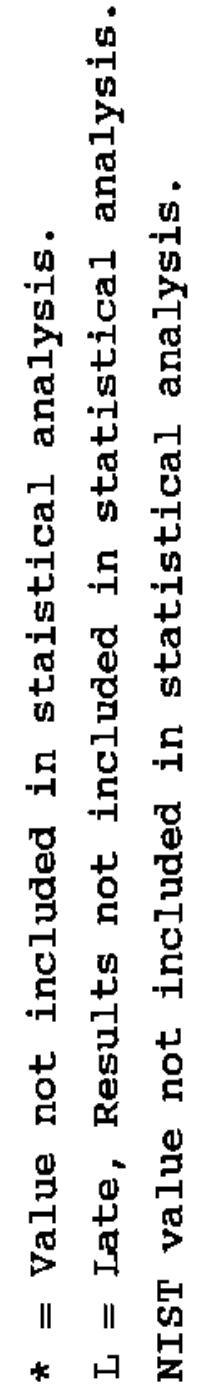




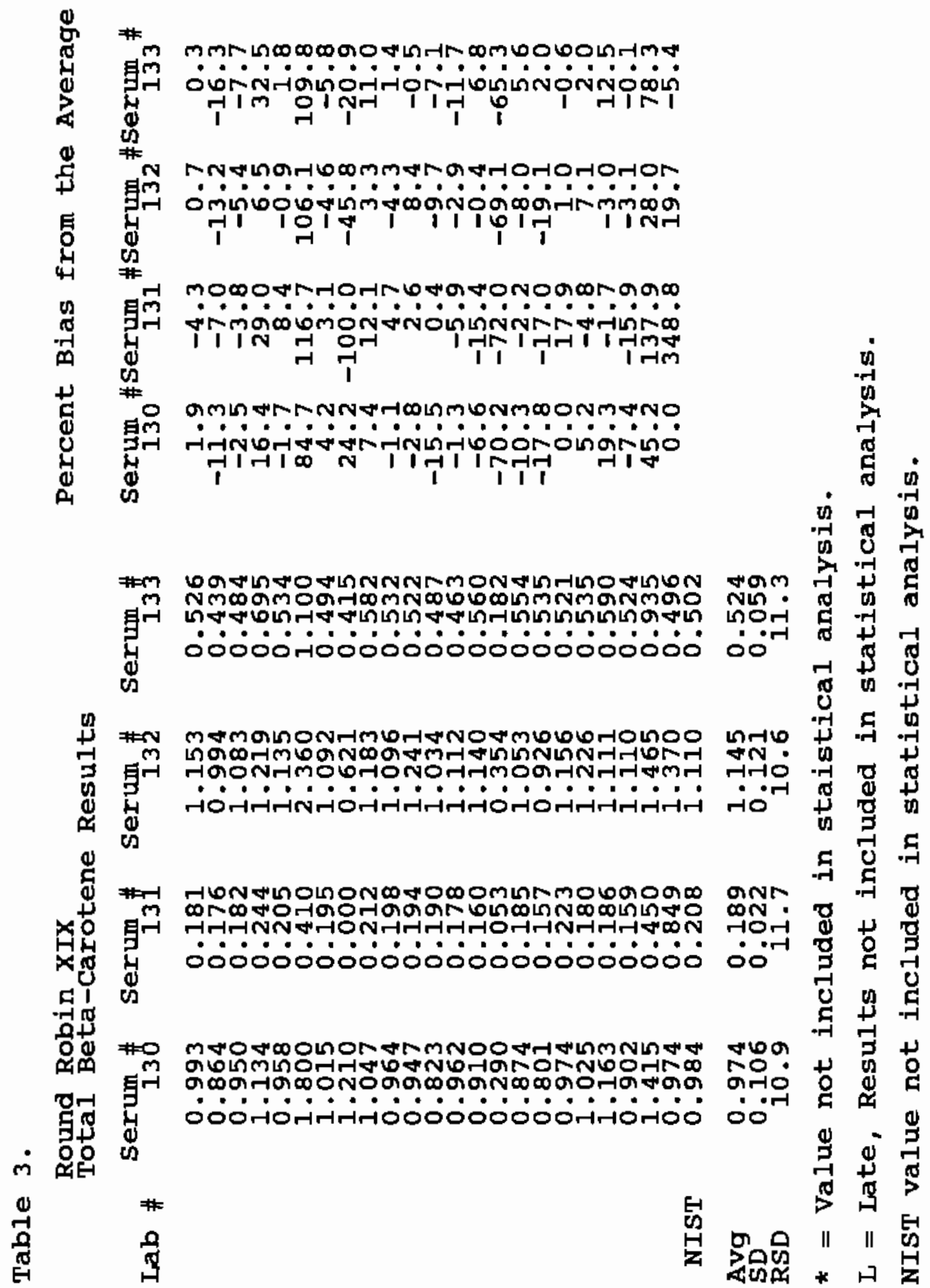




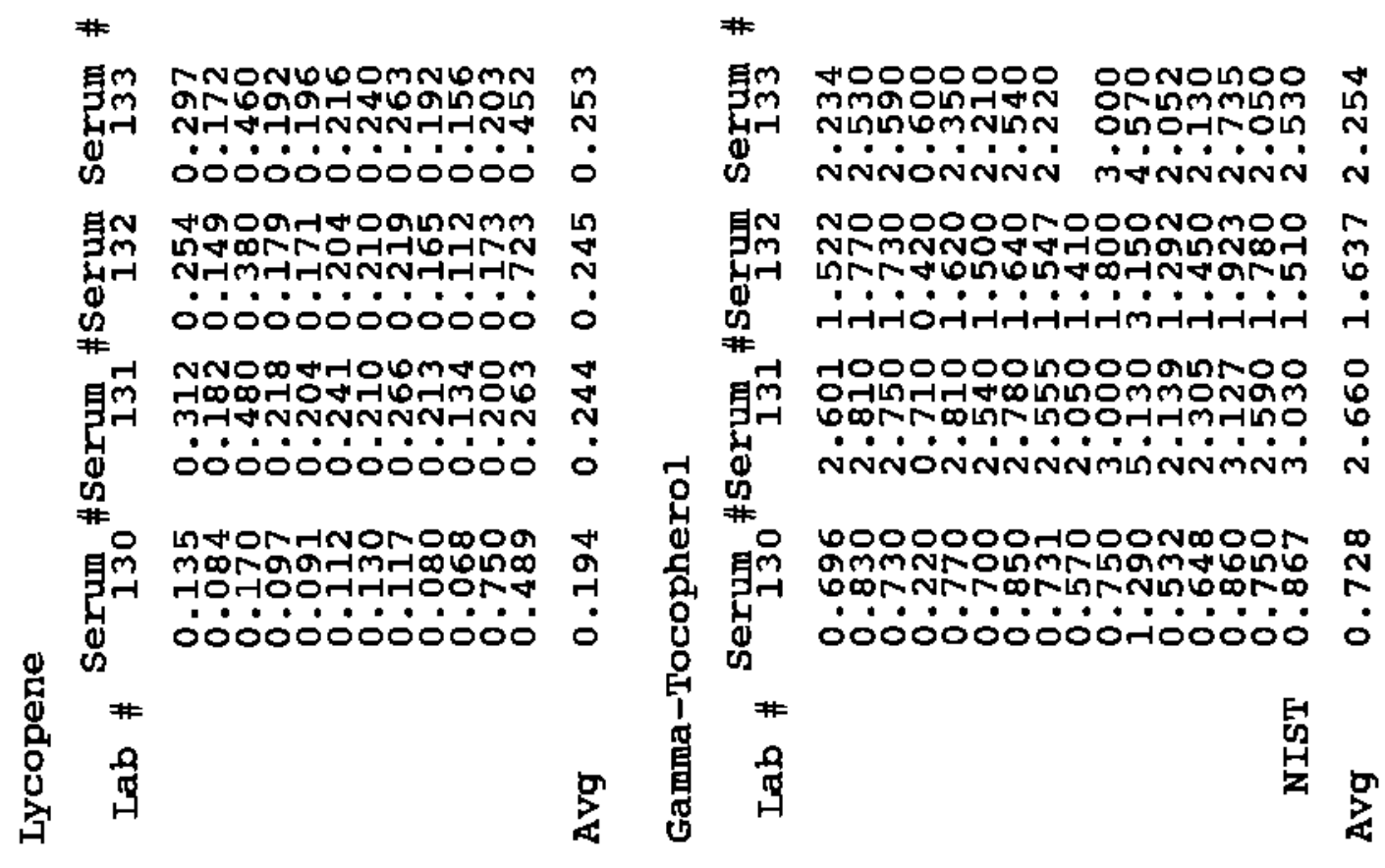

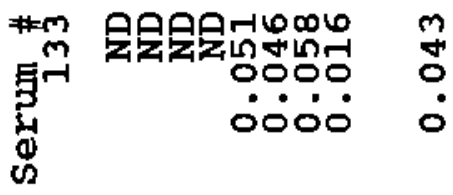

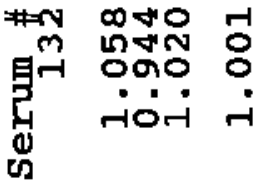

\#- $\infty$ No $\infty$

\#

म

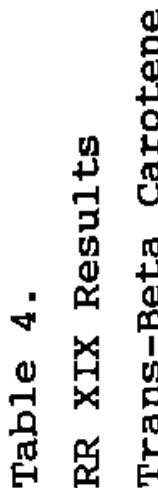

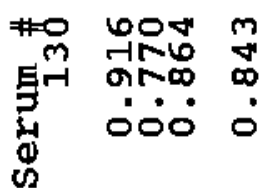

+

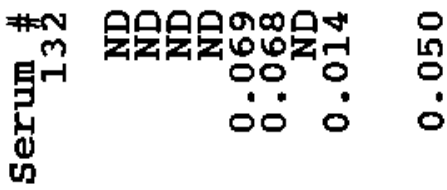

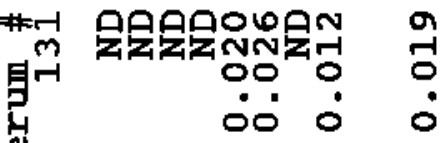

ต

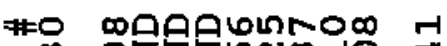

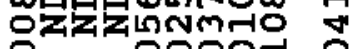
: 0.00ㅇ.

心

\#

密

gr 


\section{Interlaboratory Precision vs Time Retinol}
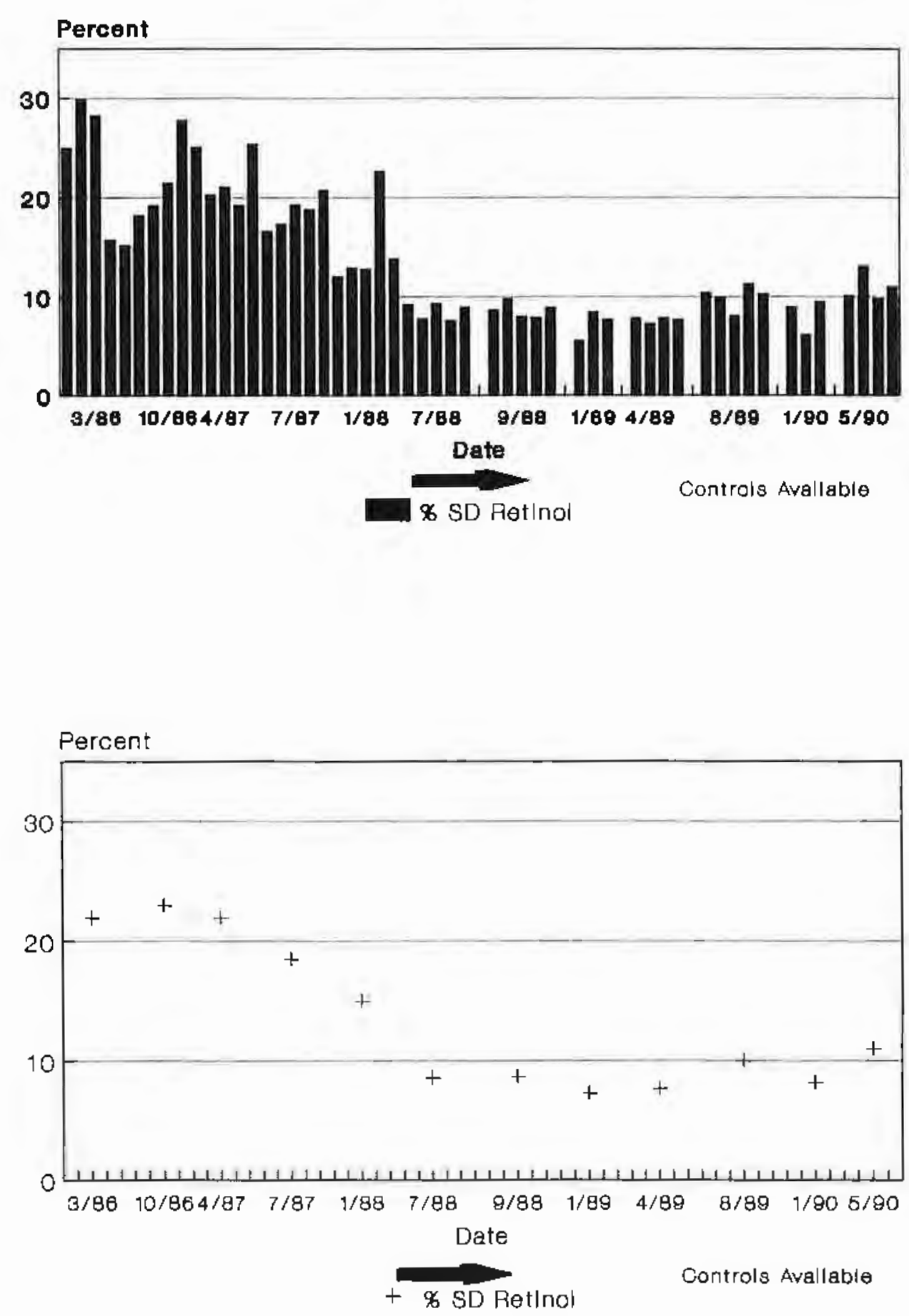


\section{Interlaboratory Precision vs Time Alpha-Tocopherol}

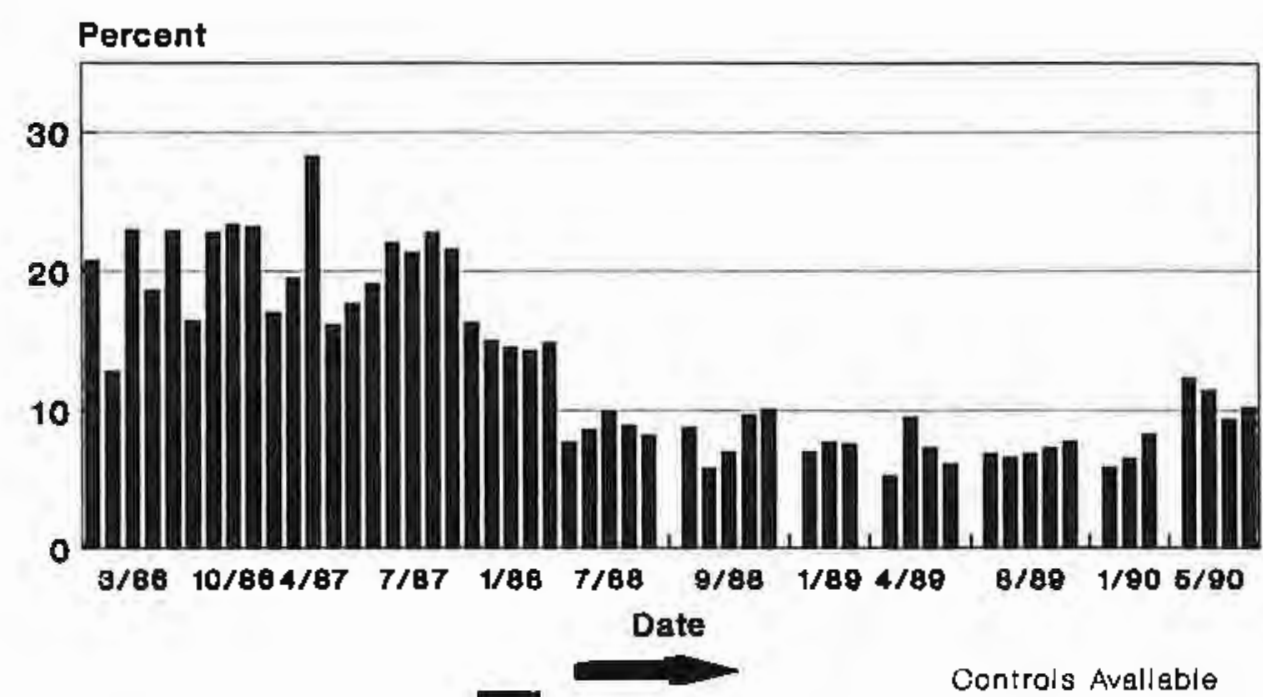

क SD Tocopherol

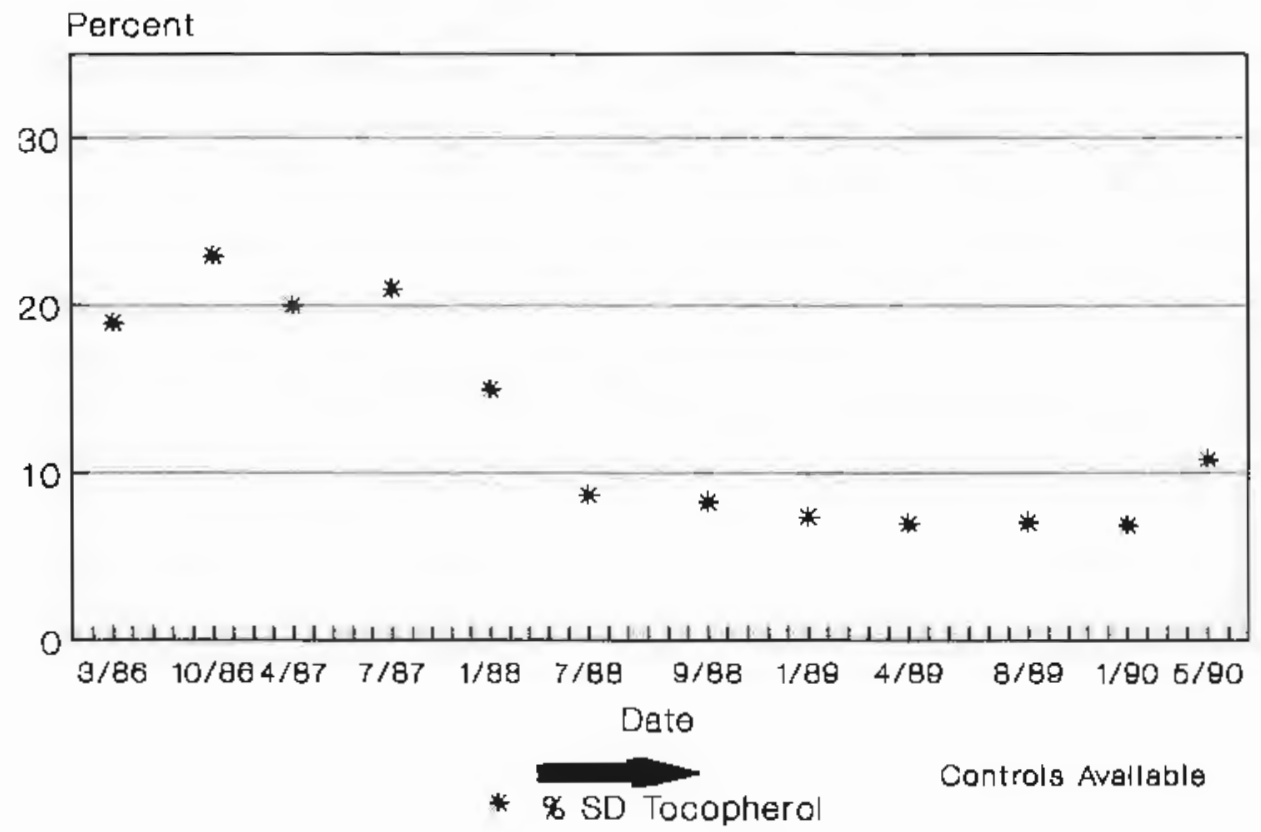




\section{Interlaboratory Precision vs Time Beta-Carotene}

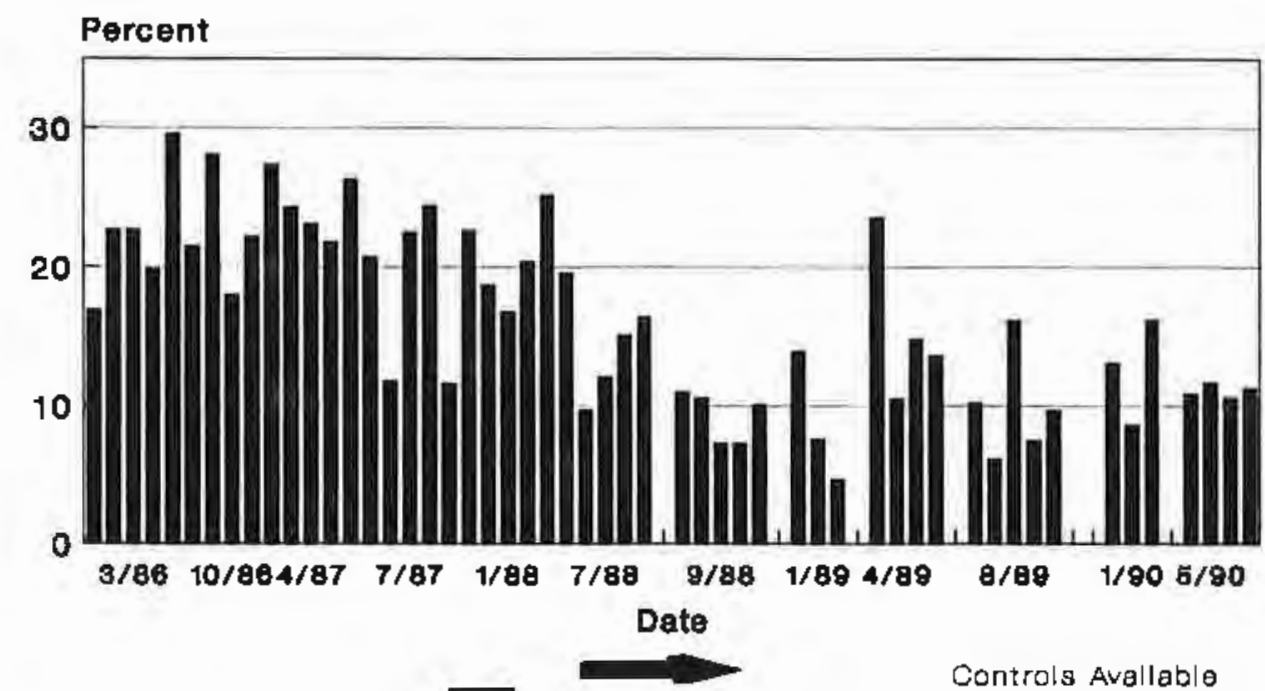

क SD Beta-Carotene

Controls Avallable

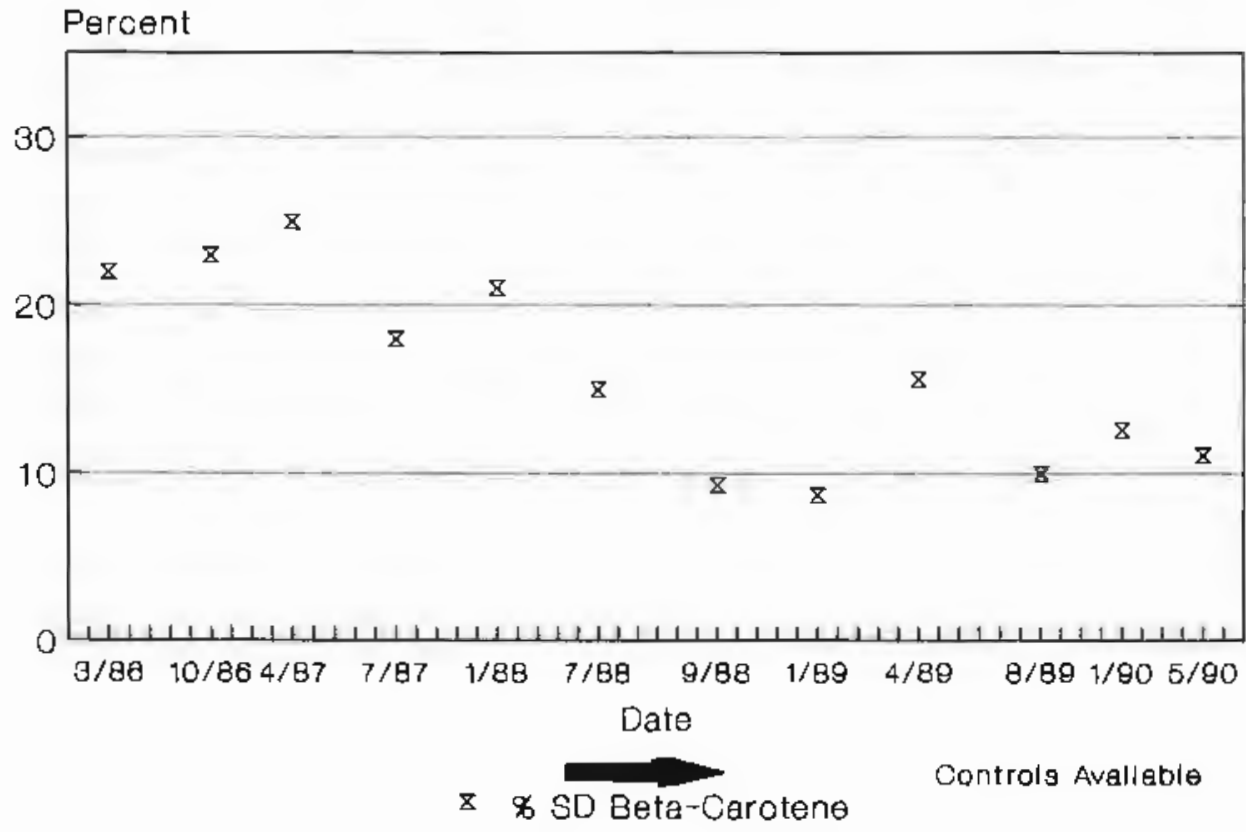




\section{Appendix F. Updated “All-Lab Report” for RR19}

The following four pages are an updated version of an "All-Lab" report for RR19. This report has three parts:

- pages 1 and 2 list results for all analytes reported at least twice, counting both participants and NIST analysts.

- $\quad$ page 3 provides a legend for pages 1 and 2 .

- $\quad$ page 4 summarizes each participants' performance for retinol, $\alpha$-tocopherol, and total $\beta$-carotene. These summaries are compatible with the percent bias evaluation advice given in the RR19 Report. However, the current bias summaries are estimated relative to the median of all reported values for each analyte in each serum rather than to the trimmed average ("Avg") used in the original and detailed in Appendix E. The original reference values were estimated from on-time results, with subjective exclusion of results deemed nonrepresentative.

To ensure confidentiality, the laboratory identifiers used in this "All-Lab Report" have been altered from those used in RR19. The only attributed results are those reported by NIST. The NIST results are not used in the assessment of the consensus summary results of the study.

Note: The results designated NISTa in this updated All-Lab report are designated NIST in the Tables of Appendix E. 


\section{Round Robin XIX Laboratory Results}

\begin{tabular}{|c|c|c|c|c|c|c|c|c|c|c|c|c|c|c|c|c|}
\hline \multirow[b]{2}{*}{$a b$} & \multicolumn{4}{|c|}{ Total Retinol } & \multicolumn{4}{|c|}{ Retinyl Palmitate } & \multicolumn{4}{|c|}{ a-Tocopherol } & \multicolumn{4}{|c|}{ ү/ß-Tocopherol } \\
\hline & 130 & 131 & 132 & 133 & 130 & 131 & 132 & 133 & 130 & 131 & 132 & 133 & 130 & 131 & 132 & 133 \\
\hline FSV-BA & 0.234 & 0.536 & 0.297 & 0.786 & & & & & 3.76 & 6.48 & 4.53 & 11.09 & & & & \\
\hline FSV-BD & 231 & 0.514 & 0.282 & 0.756 & & & & & 3.65 & 6.43 & 4.66 & 11.90 & 0.73 & 2.75 & 1.73 & 2.59 \\
\hline FSV-BE & 236 & 0.482 & 0.292 & 0.748 & & & & & 3.84 & 6.28 & 4.67 & 11.12 & 0.85 & 2.78 & 1.64 & 2.54 \\
\hline FSV-BH & 211 & 0.499 & 0.281 & 0.730 & nd & nd & nd & $n d$ & 3.88 & 6.82 & 4.54 & 11.41 & 0.73 & 2.56 & 1.55 & 2.22 \\
\hline FSV-BI & 230 & 0.488 & 0.273 & 0.729 & $n d$ & $n d$ & $n d$ & nd & 3.82 & 6.57 & 4.55 & 11.91 & .83 & 2.81 & 1.77 & 2.53 \\
\hline FSV-BJ & 218 & 0.518 & 0.288 & 0.712 & & & & & 3.83 & 7.02 & 4.75 & 12.21 & 0.86 & 3.13 & 1.92 & 2.74 \\
\hline FSV-BK & 215 & 0.506 & 0.276 & 0.750 & & & & & 3.74 & 6.63 & 4.46 & 12.12 & & & & \\
\hline FSV-BL & 204 & 0.451 & 0.270 & 0.695 & & & & & 4.57 & 7.88 & 5.39 & 14.31 & 1.29 & 5.13 & 3.15 & 4.5 \\
\hline FSV-BM & 205 & 0.526 & 0.291 & 0.748 & & & & & 3.80 & 7.30 & 4.90 & 12.40 & & & & \\
\hline FSV-BY & 0.188 & 0.425 & 0.244 & 0.745 & 0.008 & nd & nd & nd & 3.73 & 6.55 & 4.43 & 11.48 & 0.70 & 2.60 & 1.52 & 2.23 \\
\hline FSV-BZ & & & & & & & & & 6.26 & 7.65 & 4.84 & 11.65 & & & & \\
\hline FSV-CA & 192 & 0.478 & 0.309 & 0.754 & & & & & 3.84 & 6.89 & 5.02 & 13.09 & & & & \\
\hline FSV-CH & 255 & 0.610 & 0.290 & 0.765 & & & & & 4.81 & 7.70 & 5.84 & 13.70 & & & & \\
\hline FSV-CJ & 0.243 & 0.569 & 0.292 & 0.847 & & & & & 4.17 & 7.12 & 4.99 & 12.69 & & & & \\
\hline FSV-CM & & & & & & & & & 3.40 & 7.20 & 4.60 & 13.60 & & & & \\
\hline FSV-CN & na & 0.484 & 0.315 & 0.657 & & & & & 3.55 & 6.73 & 4.38 & 12.14 & 0.77 & 2.81 & 1.62 & 2.35 \\
\hline FSV-CO & 0.222 & 0.523 & 0.290 & 0.731 & & & & & 3.66 & 6.73 & 4.55 & 11.71 & & & & \\
\hline FSV-CQ & 0.264 & 0.455 & 0.299 & 0.801 & & & & & 3.57 & 6.53 & 4.63 & 12.70 & & & & \\
\hline FSV-CS & 238 & 0.529 & 0.303 & 0.745 & & & & & 2.81 & 4.84 & 3.52 & 9.12 & & & & \\
\hline FSV-CV & 233 & 0.526 & 0.292 & 0.775 & & & & & 4.47 & 6.37 & 4.85 & 10.10 & 0.75 & 2.59 & 1.78 & 2.05 \\
\hline FSV-CY & 230 & 0.510 & 0.290 & 0.780 & & & & & 3.83 & 6.51 & 4.65 & 11.95 & 0.57 & 2.05 & 1.41 & 1.8 \\
\hline FSV-DC & 208 & 0.504 & 0.274 & 0.724 & $n d$ & $n$ & $n d$ & $n d$ & & & & & & & & \\
\hline FSV-DE & & & & & & & & & 3.75 & 6.62 & 4.52 & 11.93 & 0.70 & 2.54 & 1.50 & 2.2 \\
\hline FSV-DG & 247 & 0.571 & 301 & 0 & & & & & 4.01 & 6.46 & 4.37 & 12.45 & & & & \\
\hline FSV-DH & 235 & 0.578 & 0.332 & 0.875 & 0.056 & 0.020 & 0.069 & 0.051 & 3.26 & 6.47 & 4.33 & 12.96 & 0.53 & 2.14 & 1.29 & 2.0 \\
\hline FSV-DL & 260 & 0.489 & 0.288 & 0.739 & & & & & & & & & & & & \\
\hline FSV-DN & 420 & 0.990 & 0.540 & 350 & & & & & 1.60 & 2.80 & 1.90 & 4.60 & 0.22 & 0.71 & 0.42 & 0.6 \\
\hline FSV-DO & 196 & 359 & 0.216 & 522 & & & & & 13.04 & 7.41 & 7.21 & 7.31 & & & & \\
\hline FSV-DY & 424 & 442 & 248 & 614 & & & & & & & & & & & & \\
\hline FSV-DZ & 248 & 686 & 311 & 695 & & & & & 3.91 & 4.52 & 3.96 & 9.61 & & & & \\
\hline FSV-EB & 235 & 0.530 & 292 & 775 & & & & & 3.75 & 6.18 & 4.21 & 11.08 & & & & \\
\hline FSV-ED & 222 & 0.527 & 285 & 757 & & & & & 3.79 & 6.90 & 4.63 & 11.81 & & & & \\
\hline FSV-EF & 250 & 0.550 & 330 & 840 & & & & & 3.20 & 6.30 & 4.20 & 11.60 & 0.75 & 3.00 & 1.80 & 3.0 \\
\hline FSV-EJ & 0.188 & 0.229 & 263 & 575 & & & & & 1.92 & 2.05 & 1.12 & 2.95 & & & & \\
\hline FSV-EO & 0.177 & 0.402 & 234 & 0.601 & & & & & & & & & & & & \\
\hline FSV-EV & 0.238 & 0.661 & 0.363 & 0.926 & & & & & 3.80 & 7.40 & 4.80 & 12.80 & & & & \\
\hline FSV-EY & 0.223 & 0.556 & 0.321 & 0.719 & & & & & 3.58 & 6.85 & 4.89 & 11.23 & & & & \\
\hline FSV-FA & 0.252 & 0.452 & 0.303 & 0.668 & 0.01 & 0.012 & 0.014 & 0.01 & 2.58 & 5.17 & 2.06 & 9.67 & 0.65 & 2.31 & 1.45 & 2.1 \\
\hline FSV-FQ & 0.257 & 0.482 & 0.318 & 0.674 & 0.025 & 0.026 & 0.068 & 0.04 & & & & & & & & \\
\hline FSV-FV & 0.279 & 0.560 & 0.318 & 0.867 & 0.037 & $n q$ & $n q$ & 0.058 & & & & & & & & \\
\hline $\mathrm{n}$ & 36 & 37 & 37 & 37 & 4 & 3 & 3 & & 34 & 34 & 34 & 34 & 15 & 15 & 15 & 15 \\
\hline Min & 0.177 & 0.229 & 216 & 0.522 & 008 & & 4 & & 50 & 2.05 & 1.12 & 2.95 & 22 & 0.71 & 0.42 & $0.6 C$ \\
\hline Mean & 0.239 & 0.519 & 298 & 6 & & & & & 3.98 & 6.39 & 4.44 & 11.25 & 73 & 2.66 & 1.64 & 2.3 \\
\hline Max & 0.424 & 0.990 & 0.540 & & 0.056 & & & 0.058 & 13.04 & 7.88 & 7.21 & 14.31 & 1.29 & 5.13 & 3.15 & 4.5 \\
\hline SD & 0.051 & 0.112 & 0.049 & 0.129 & 0.020 & 0.007 & 0.031 & 0.019 & 1.78 & 1.24 & 1.05 & 2.34 & 0.22 & 0.89 & 0.55 & 0.8 \\
\hline $\mathrm{CV}$ & 21 & 22 & 17 & 17 & 64 & 36 & 63 & 43 & 45 & 19 & 24 & 21 & 30 & 33 & 33 & 34 \\
\hline NISTa & 0.212 & 0.524 & 0.307 & 0.820 & & & & & 3.32 & 6.75 & 4.43 & 11.46 & 0.87 & 3.03 & 1.51 & 2.53 \\
\hline Median & 34 & 514 & 292 & 0.748 & & & & & 3.78 & 6.60 & 4.58 & 11.86 & .73 & 2.60 & .62 & 2.2 \\
\hline eSD & 0.026 & 0.053 & 0.024 & 0.053 & & & & & 0.24 & 0.44 & 0.35 & 1.11 & 0.12 & 0.31 & 0.24 & 0.4 \\
\hline eCV & 11 & 10 & 8 & 7 & & & & & 6 & 7 & 8 & 9 & 17 & 12 & 15 & 2 \\
\hline
\end{tabular}




\section{Round Robin XIX Laboratory Results}

\begin{tabular}{|c|c|c|c|c|c|c|c|c|c|c|c|c|c|c|c|c|}
\hline \multirow[b]{2}{*}{ Lab } & \multicolumn{4}{|c|}{ Total $\beta$-Carotene } & \multicolumn{4}{|c|}{ trans- $\beta$-Carotene } & \multicolumn{4}{|c|}{ Total cis- $\beta$-Carotene } & \multicolumn{4}{|c|}{ Total Lycopene } \\
\hline & 130 & 131 & 132 & 133 & 130 & 131 & 132 & 133 & 130 & 131 & 132 & 133 & 130 & 131 & 132 & 133 \\
\hline FSV-BA & 0.909 & 0.201 & 1.086 & 0.522 & & & & & & & & & & & & \\
\hline FSV-BD & & & & & & & & & & & & & & & & \\
\hline FSV-BE & & & & & & & & & & & & & & & & \\
\hline FSV-BH & 0.962 & 0.178 & 1.112 & 0.463 & 0.916 & 0.168 & 1.058 & 0.441 & 0.046 & 0.010 & 0.054 & 0.022 & 0.112 & 0.241 & 0.204 & 0.216 \\
\hline FSV-BI & 0.864 & 0.176 & 0.994 & 0.439 & & & & & & & & & 0.084 & 0.182 & 0.149 & 0.172 \\
\hline FSV-BJ & 1.025 & 0.180 & 1.226 & 0.535 & & & & & & & & & 0.068 & 0.134 & 0.112 & 0.156 \\
\hline FSV-BK & & & & & & & & & & & & & & & & \\
\hline FSV-BL & & & & & & & & & & & & & & & & \\
\hline FSV-BM & & & & & & & & & & & & & & & & \\
\hline FSV-BY & 0.993 & 0.181 & 1.153 & 0.526 & & & & & & & & & 0.135 & 0.312 & 0.254 & 0.297 \\
\hline FSV-BZ & & & & & & & & & & & & & & & & \\
\hline FSV-CA & & & & & & & & & & & & & & & & \\
\hline FSV-CH & 1.415 & 0.450 & 1.465 & 0.935 & & & & & & & & & & & & \\
\hline FSV-CJ & 0.950 & 0.182 & 1.083 & 0.484 & & & & & & & & & & & & \\
\hline FSV-CM & & & & & & & & & & & & & & & & \\
\hline FSV-CN & 1.015 & 0.195 & 1.092 & 0.494 & & & & & & & & & 0.097 & 0.218 & 0.179 & 0.192 \\
\hline FSV-CO & 0.958 & 0.205 & 1.135 & 0.534 & & & & & & & & & & & & \\
\hline FSV-CQ & 1.210 & nd & 0.621 & 0.415 & & & & & & & & & & & & \\
\hline FSV-CS & & & & & & & & & & & & & & & & \\
\hline FSV-CV & 1.163 & 0.186 & 1.111 & 0.590 & & & & & & & & & 0.108 & 0.200 & 0.173 & 0.203 \\
\hline FSV-CY & 0.910 & 0.160 & 1.140 & 0.560 & & & & & & & & & 0.130 & 0.210 & 0.210 & 0.240 \\
\hline FSV-DC & 0.964 & 0.198 & 1.096 & 0.532 & & & & & & & & & 0.091 & 0.204 & 0.171 & 0.196 \\
\hline FSV-DE & & & & & & & & & & & & & & & & \\
\hline FSV-DG & 1.047 & 0.212 & 1.183 & 0.582 & & & & & & & & & & & & \\
\hline FSV-DH & 0.874 & 0.185 & 1.053 & 0.554 & 0.770 & 0.167 & 0.944 & 0.492 & 0.104 & 0.018 & 0.109 & 0.062 & 0.117 & 0.266 & 0.219 & 0.263 \\
\hline FSV-DL & 1.067 & 0.166 & 1.138 & 0.527 & & & & & & & & & & & & \\
\hline FSV-DN & 1.800 & 0.410 & 2.360 & 1.100 & & & & & & & & & 0.170 & 0.480 & 0.380 & 0.460 \\
\hline FSV-DO & 1.134 & 0.244 & 1.219 & 0.695 & & & & & & & & & & & & \\
\hline FSV-DY & 0.290 & 0.053 & 0.354 & 0.182 & & & & & & & & & & & & \\
\hline FSV-DZ & & & & & & & & & & & & & & & & \\
\hline FSV-EB & 0.902 & 0.159 & 1.110 & 0.524 & & & & & & & & & & & & \\
\hline FSV-ED & 0.823 & 0.190 & 1.034 & 0.487 & & & & & & & & & & & & \\
\hline FSV-EF & & & & & & & & & & & & & & & & \\
\hline FSV-EJ & 1.586 & 0.176 & 1.370 & 0.496 & & & & & & & & & 0.489 & 0.263 & 0.723 & 0.452 \\
\hline FSV-EO & 0.947 & 0.194 & 1.241 & 0.522 & & & & & & & & & & & & \\
\hline FSV-EV & & & & & & & & & & & & & & & & \\
\hline FSV-EY & & & & & & & & & & & & & & & & \\
\hline FSV-FA & 0.974 & 0.223 & 1.156 & 0.521 & & & & & & & & & 0.080 & 0.213 & 0.165 & 0.192 \\
\hline FSV-FQ & 0.801 & 0.157 & 0.926 & 0.535 & & & & & & & & & & & & \\
\hline FSV-FV & & & & & & & & & & & & & & & & \\
\hline $\mathrm{n}$ & 25 & 24 & 25 & 25 & 2 & 2 & 2 & 2 & 2 & 2 & 2 & 2 & 12 & 12 & 12 & 12 \\
\hline Min & 0.290 & 0.053 & 0.354 & 0.182 & 0.770 & 0.167 & 0.944 & 0.441 & 0.046 & 0.010 & 0.054 & 0.022 & 0.068 & 0.134 & 0.112 & 0.156 \\
\hline Mean & 1.023 & 0.203 & 1.138 & 0.550 & 0.843 & 0.168 & 1.001 & 0.467 & 0.075 & 0.014 & 0.082 & 0.042 & 0.140 & 0.244 & 0.245 & 0.253 \\
\hline Max & 1.800 & 0.450 & 2.360 & 1.100 & 0.916 & 0.168 & 1.058 & 0.492 & 0.104 & 0.018 & 0.109 & 0.062 & 0.489 & 0.480 & 0.723 & 0.460 \\
\hline SD & 0.280 & 0.078 & 0.333 & 0.167 & & & & & & & & & 0.113 & 0.087 & 0.165 & 0.102 \\
\hline CV & 27 & 39 & 29 & 30 & & & & & & & & & 81 & 36 & 67 & 40 \\
\hline $\mathrm{NISTa}$ & \begin{tabular}{|l}
0.984 \\
\end{tabular} & 0.208 & 1.110 & 0.502 & 0.864 & 0.184 & 1.020 & 0.432 & \begin{tabular}{|l|l}
0.120 \\
\end{tabular} & 0.024 & 0.090 & 0.070 & & & & \\
\hline Median & 0.964 & 0.186 & 1.112 & 0.526 & & & & & & & & & 0.110 & 0.216 & 0.192 & 0.210 \\
\hline eSD & 0.123 & 0.021 & 0.087 & 0.047 & & & & & & & & & 0.033 & 0.044 & 0.040 & 0.050 \\
\hline eCV & 13 & 11 & 8 & 9 & & & & & & & & & 30 & 20 & 21 & 24 \\
\hline
\end{tabular}




\section{Round Robin XIX Laboratory Results}

\section{Table Legend}

\begin{tabular}{r|l} 
Symbol & \\
\hline$n a$ & Not available (not reported by participant) \\
$n d$ & Not detected (not reported or reported as '0', 'not determined', etc.) \\
$<\mathrm{x}$ & Value less than $\mathrm{x}$ \\
italics & Value calculated from reported results \\
$\mathrm{n}$ & Number of non-NIST laboratories reporting quantitative results \\
Min & Minimum non-NIST reported value. \\
Mean & Average over all non-NIST reported values. \\
Max & Maximum non-NIST reported value. \\
SD & Standard deviation over all non-NIST values. \\
$\mathrm{CV}$ & Coefficient of Variation (\% relative standard deviation): 100*SD/Mean \\
& \\
Median & Median over all non-NIST reported values \\
eSD & Robust estimate of SD based on the adjusted median absolute difference from the median (MADe) \\
eCV & Robust estimate of CV, 100*eSD/Median
\end{tabular}




\section{Round Robin XIX Laboratory Results}

\%Bias Summary

\begin{tabular}{|c|c|c|c|}
\hline Lab & TR & aT & $\mathrm{bC}$ \\
\hline FSV-BA & $3 \pm 2$ & $-2 \pm 3$ & $0 \pm 6$ \\
\hline FSV-BD & $-1 \pm 2$ & $-1 \pm 2$ & \\
\hline FSV-BE & $-1 \pm 3$ & $-2 \pm 4$ & \\
\hline FSV-BH & $-5 \pm 3$ & $0 \pm 3$ & $-4 \pm 6$ \\
\hline FSV-BI & $-4 \pm 2$ & $0 \pm 1$ & $-11 \pm 5$ \\
\hline FSV-BJ & $-3 \pm 3$ & $4 \pm 2$ & $4 \pm 6$ \\
\hline FSV-BK & $-4 \pm 4$ & $0 \pm 2$ & \\
\hline FSV-BL & $-10 \pm 3$ & $20 \pm 1$ & \\
\hline FSV-BM & $-3 \pm 7$ & $6 \pm 4$ & \\
\hline FSV-BY & $-13 \pm 9$ & $-2 \pm 1$ & $1 \pm 3$ \\
\hline FSV-BZ & & $21 \pm 30$ & \\
\hline FSV-CA & $-5 \pm 10$ & $7 \pm 4$ & \\
\hline $\mathrm{FSV}-\mathrm{CH}$ & $7 \pm 9$ & $22 \pm 7$ & $75 \pm 49$ \\
\hline FSV-CJ & $7 \pm 6$ & $9 \pm 1$ & $-3 \pm 3$ \\
\hline FSV-CM & & $4 \pm 11$ & \\
\hline $\mathrm{FSV}-\mathrm{CN}$ & $-3 \pm 10$ & $-1 \pm 4$ & $1 \pm 6$ \\
\hline FSV-CO & $-2 \pm 3$ & $-1 \pm 2$ & $3 \pm 5$ \\
\hline FSV-CQ & $3 \pm 10$ & $0 \pm 5$ & $-13 \pm 35$ \\
\hline FSV-CS & $2 \pm 2$ & $-25 \pm 2$ & \\
\hline $\mathrm{FSV}-\mathrm{CV}$ & $1 \pm 2$ & $2 \pm 14$ & $8 \pm 10$ \\
\hline FSV-CY & $0 \pm 3$ & $1 \pm 1$ & $-3 \pm 9$ \\
\hline FSV-DC & $-6 \pm 4$ & & $2 \pm 4$ \\
\hline FSV-DE & & $0 \pm 1$ & \\
\hline FSV-DG & $7 \pm 3$ & $1 \pm 5$ & $10 \pm 3$ \\
\hline FSV-DH & $11 \pm 7$ & $-3 \pm 10$ & $-2 \pm 6$ \\
\hline FSV-DL & $1 \pm 7$ & & $1 \pm 9$ \\
\hline FSV-DN & $84 \pm 6$ & $-59 \pm 2$ & $107 \pm 15$ \\
\hline FSV-DO & $-26 \pm 7$ & $69 \pm 124$ & $23 \pm 11$ \\
\hline FSV-DY & $9 \pm 49$ & & $-69 \pm 3$ \\
\hline FSV-DZ & $10 \pm 17$ & $-15 \pm 15$ & \\
\hline FSV-EB & $2 \pm 2$ & $-5 \pm 3$ & $-5 \pm 7$ \\
\hline FSV-ED & $-1 \pm 3$ & $1 \pm 2$ & $-7 \pm 7$ \\
\hline FSV-EF & $10 \pm 3$ & $-8 \pm 6$ & \\
\hline FSV-EJ & $-27 \pm 20$ & $-67 \pm 12$ & $19 \pm 33$ \\
\hline FSV-EO & $-21 \pm 2$ & & $3 \pm 6$ \\
\hline FSV-EV & $20 \pm 12$ & $6 \pm 5$ & \\
\hline FSV-EY & $2 \pm 8$ & $0 \pm 6$ & \\
\hline FSV-FA & $-3 \pm 10$ & $-32 \pm 16$ & $6 \pm 10$ \\
\hline FSV-FQ & $1 \pm 10$ & & $-12 \pm 9$ \\
\hline $\mathrm{FSV}-\mathrm{FV}$ & $13 \pm 5$ & & \\
\hline $\mathrm{NISTa}[$ & $2 \pm 8$ & $-4 \pm 6$ & $2 \pm 7$ \\
\hline
\end{tabular}

\begin{tabular}{r|l} 
Label & Definition \\
\hline Lab & Participant code \\
TR & Total Retinol \\
aT & a-Tocopherol \\
bC & Total b-Carotene
\end{tabular}

$\%$ Bias (Mean \pm SD) of individual serum biases

Mean $\mid$ Average of $\left(\mathrm{x}_{\mathrm{i}}-\right.$ Median $\left._{\mathrm{i}}\right) /$ Median $_{\mathrm{i}}$

SD Standard deviation of $\left(\mathrm{x}_{\mathrm{i}}-\right.$ Median $\left._{\mathrm{i}}\right) /$ Median $_{\mathrm{i}}$

$x_{i}$ Result for analyte in serum ${ }_{i}$

Median $_{i}$ Median of non-NIST results in serum $i$

The original analysis listed \% Bias for each result for each serum calculated relative to the "Trimmed Core Lab Average" of that analyte in the serum. The summary values reported here are the (arithmetic mean \pm standard deviation) of each laboratory's reported results for the analyte estimated relative to each serum's median value. 


\section{Appendix G. Shipping Package Inserts for RR20}

Two items were included in each package shipped to an RR20 participant:

- Cover letter. The original letter has been lost. It likely would have described the five sample materials (liquid frozen sera 138 and 139; lyophilized sera 140, 141, and 142), given guidance on reconstituting the lyophilized samples, stated that results were required by October 22, 1990, to whom they should be sent, and who to contact with technical questions. It is likely that this letter also provided details on the 1990 QA Workshop, held at NIST on November 15, 1990.

- Datasheet. The following two pages reproduce the form.

The cover letter and datasheets were attached to the shipping box. 


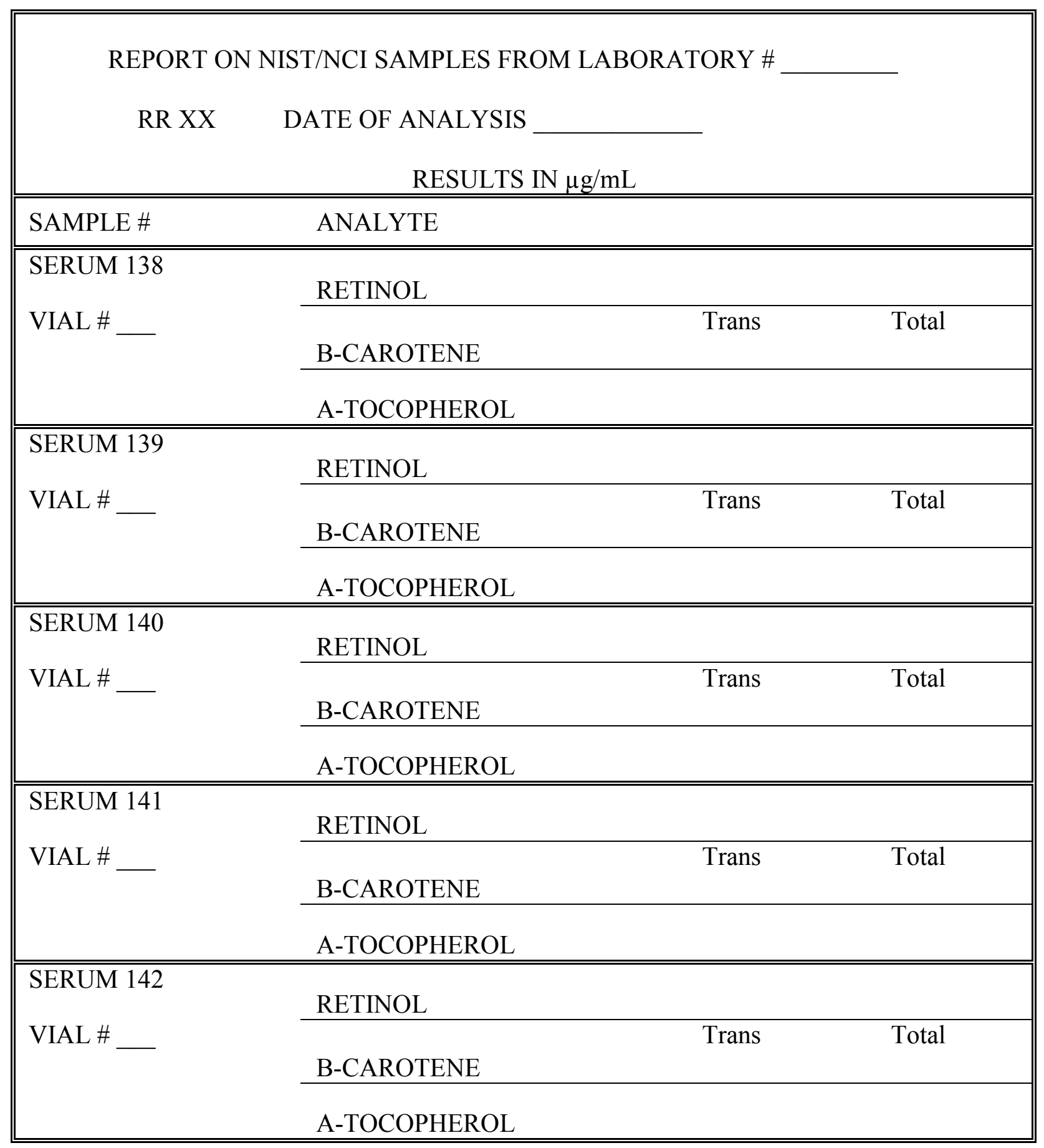

Add 1.0 mL of Distilled water to samples 140, 141, and 142. 


\begin{tabular}{|c|c|}
\hline \multicolumn{2}{|c|}{$\begin{array}{l}\text { REPORT ON NIST/NCI SAMPLES FROM LABORATORY \# } \\
\text { OPTIONAL ANALYTES } \\
\quad \text { DATE OF ANALYSIS }\end{array}$} \\
\hline \multicolumn{2}{|r|}{ RESULTS IN $\mu \mathrm{g} / \mathrm{mL}$} \\
\hline SAMPLE \# & ANALYTE \\
\hline \multirow{3}{*}{$\begin{array}{l}\text { SERUM } 138 \\
\text { VIAL \# }\end{array}$} & RETINYL PALMITATE \\
\hline & LYCOPENE \\
\hline & GAMMA-TOCOPHEROL \\
\hline \multirow{3}{*}{$\begin{array}{l}\text { SERUM } 139 \\
\text { VIAL \# }\end{array}$} & RETINYL PALMITATE \\
\hline & LYCOPENE \\
\hline & GAMMA-TOCOPHEROL \\
\hline \multirow{3}{*}{$\begin{array}{l}\text { SERUM } 140 \\
\text { VIAL \# }\end{array}$} & RETINYL PALMITATE \\
\hline & LYCOPENE \\
\hline & GAMMA-TOCOPHEROL \\
\hline \multirow{3}{*}{$\begin{array}{l}\text { SERUM } 141 \\
\text { VIAL \# }\end{array}$} & RETINYL PALMITATE \\
\hline & LYCOPENE \\
\hline & GAMMA-TOCOPHEROL \\
\hline \multirow{3}{*}{$\begin{array}{l}\text { SERUM } 142 \\
\text { VIAL \# }\end{array}$} & RETINYL PALMITATE \\
\hline & LYCOPENE \\
\hline & GAMMA-TOCOPHEROL \\
\hline
\end{tabular}




\section{Appendix H. Final Report for RR20}

There is no available record of the complete final report for RR20. The following four pages present the results and summary statistics for the samples as interpreted at the time:

- Tables 1 thru 3 list retinol, $\alpha$-tocopherol, total $\beta$-carotene;

- Tables 4 to 7 list trans- $\beta$-carotene, retinyl palmitate, lycopene, and $\gamma$-tocopherol.

It is likely that most participants reported "total lycopene" (the sum of all isomers.) Since resolution of $\gamma$-tocopherol and $\beta$-tocopherol is challenging, the results reported as $\gamma$-tocopherol can be confidently assumed to be $\gamma / \beta$-tocopherol.

Due to the complex formatting used in the tabular listings, the original laboratory codes have been deleted without replacement. However, Appendix I provides a complete listing of the RR20 results where the original codes have been altered to ensure confidentiality. Appendix I also provides more relevant summary statistics. 


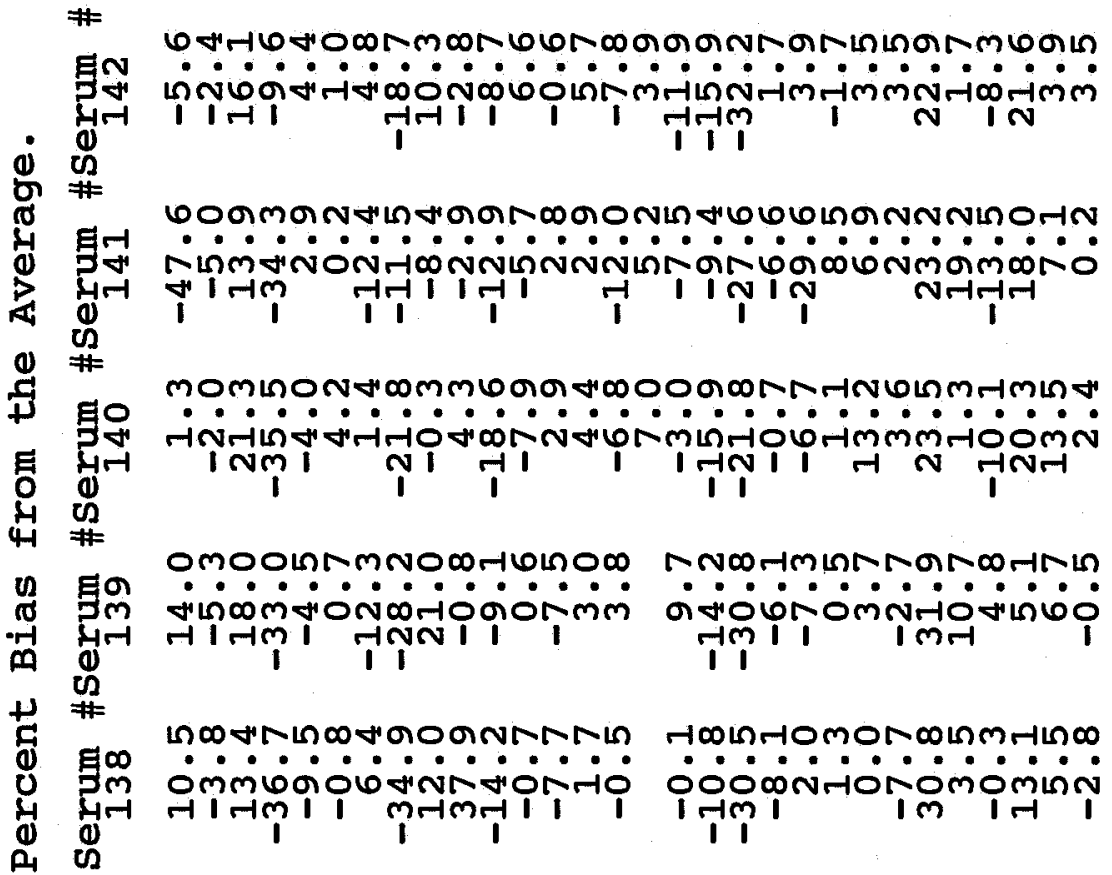

$\neq$

过

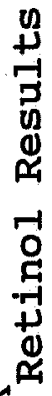

$\not$

$\therefore$

$\underset{-1}{0}$

엄

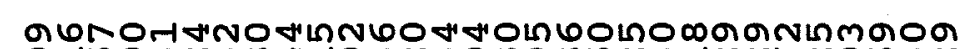

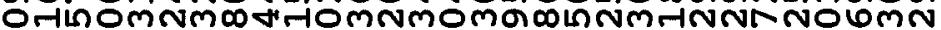

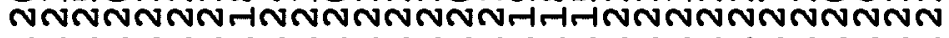
ó00000000000000000000000000 $\times$ $* *$ $x$

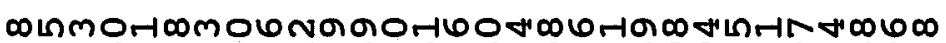
maL

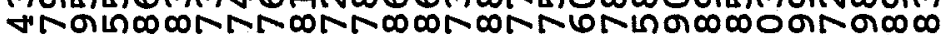

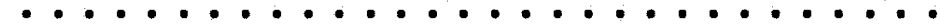
*00000000000000000000000 *

c

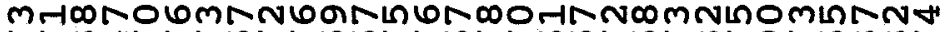

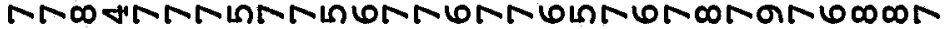
. 000000000000000000000000000000

in HoOAnOOANOHOGH

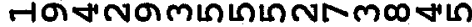

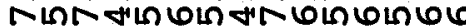

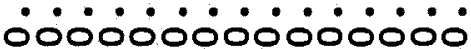
* *

HNNOOGmogntrono नMNInO सn

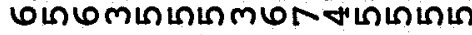

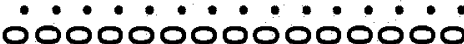
$*$ *

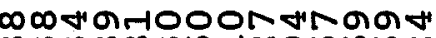

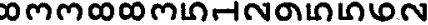

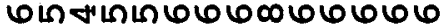
:.0.

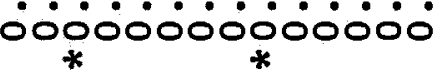

NMJitomomNHLMN

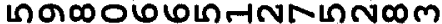

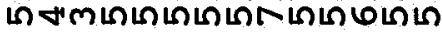
-000000000000 *

EE-
\&U
HE
'L

tima 0 NH: N Noln N io mo. $\infty$ $\infty \circ \sigma \infty$ $\therefore \dot{0}$ 000 n

बताm Nm. $M$ NOH -

Nmin กิน 000 í

moro மிษ . $100 \infty$ ○ं 


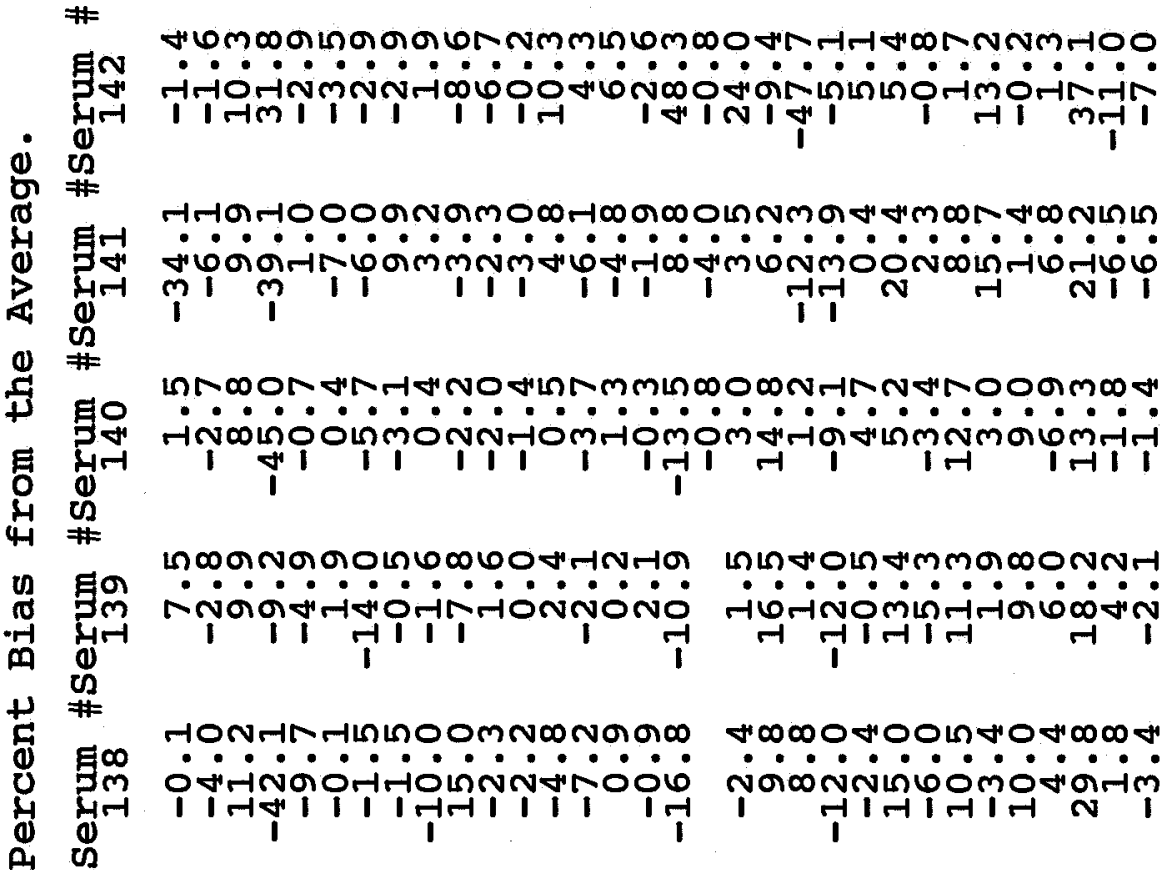

\#

Eั

Bot

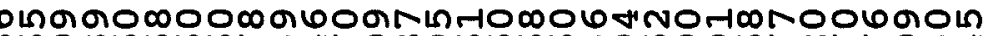

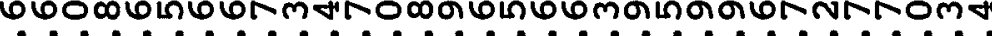

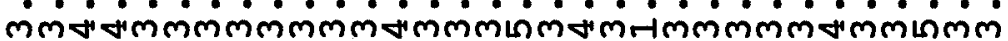
0 级

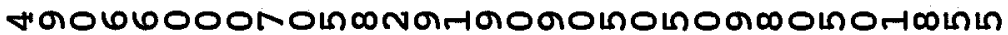

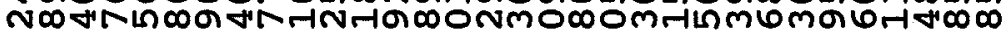
மெंம்

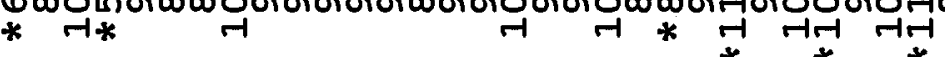

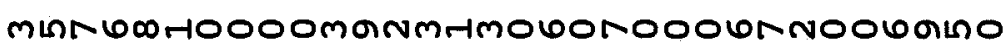
MLN

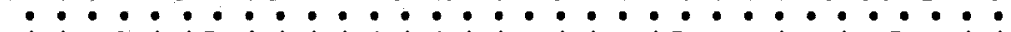
नतN

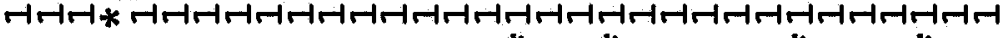

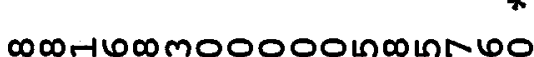

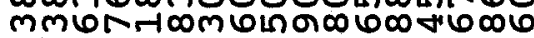

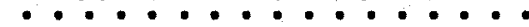

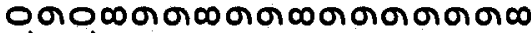
$\rightarrow+1$

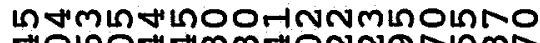

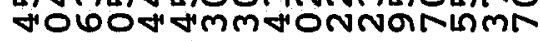

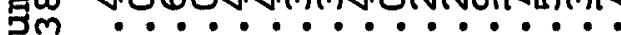

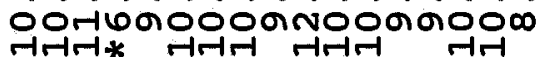

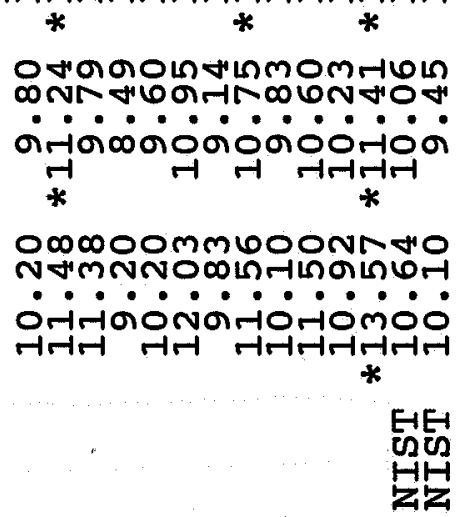

Hom w

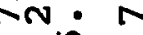
món $m$

600 सก: 6 - in ตo

$$
\text { io }
$$

மOF in $\cdot \cdot \nabla^{2}$ HO

-

non 06 . aio

$000+$ $\forall+\infty$ - $\infty$ i-

किष्ध 


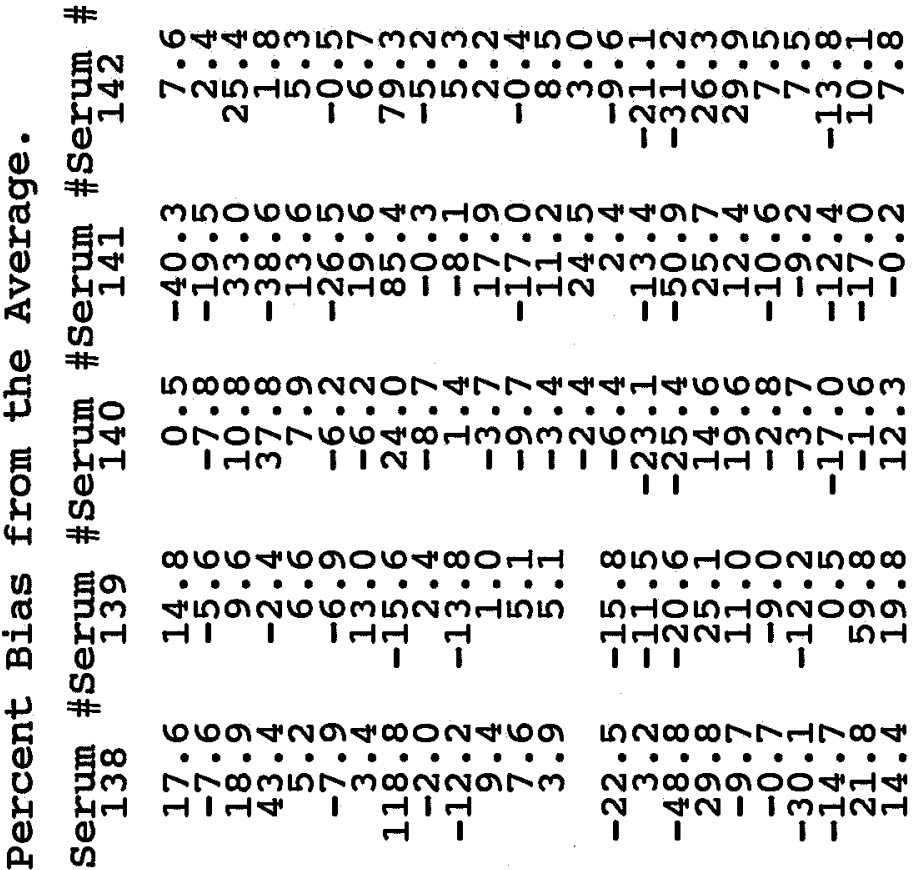

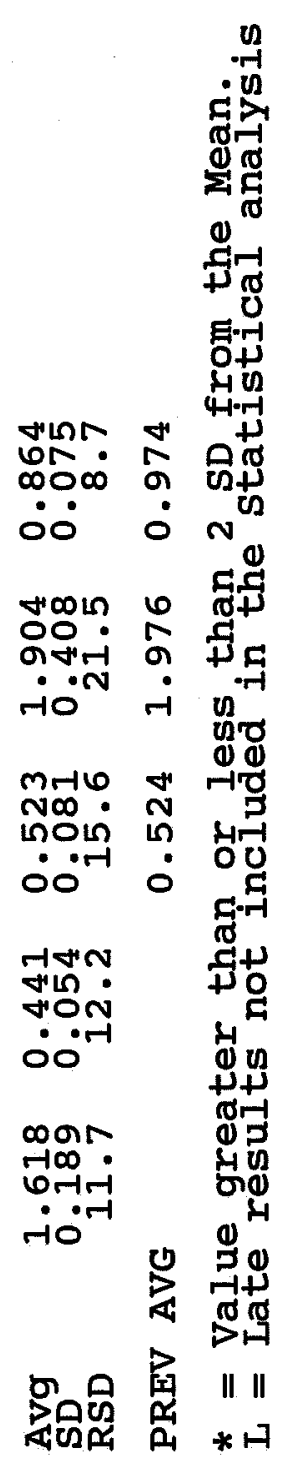



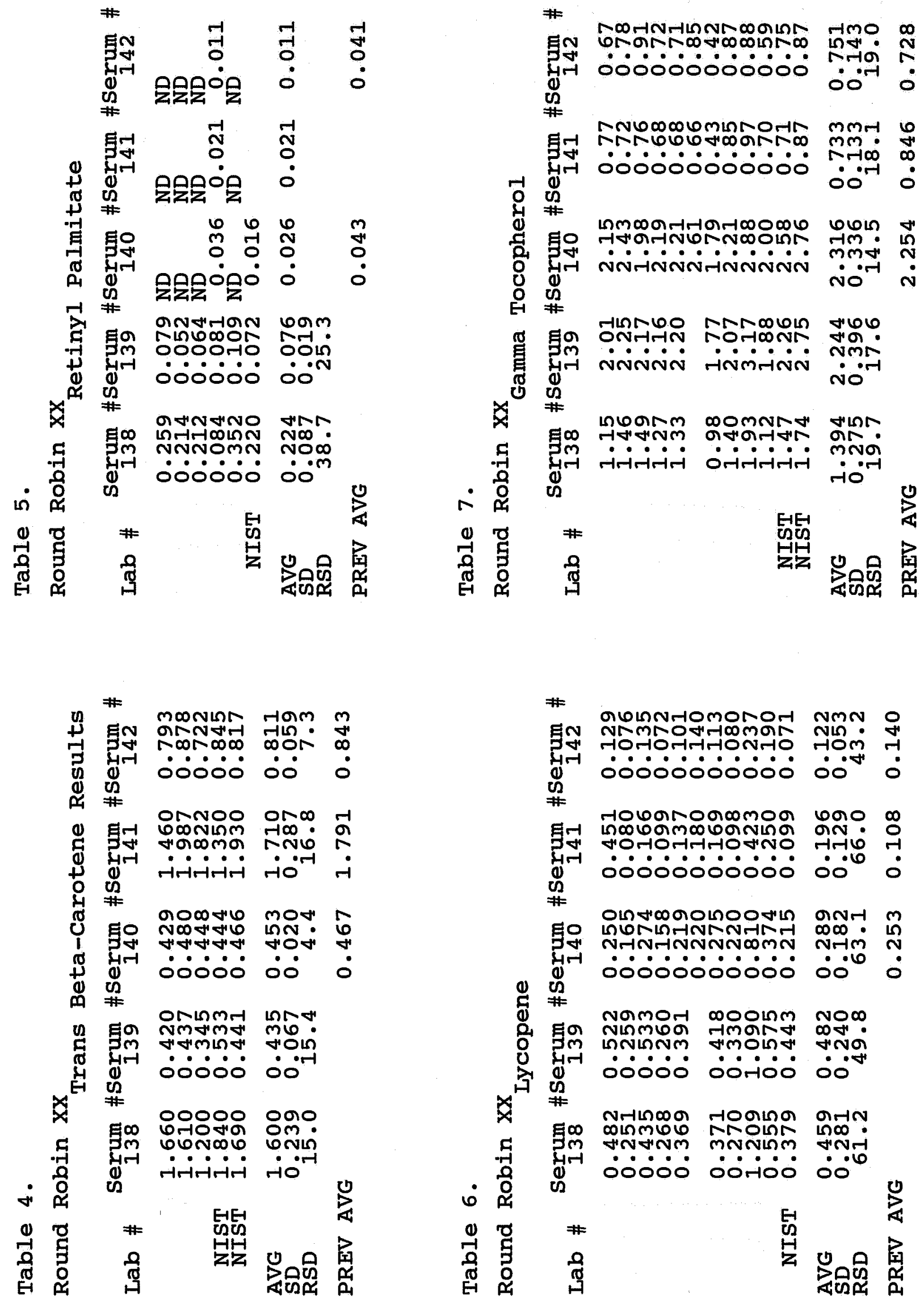


\section{Appendix I. Updated “All-Lab Report” for RR20}

The following four pages are an updated version of an "All-Lab" report for RR20. This report has three parts:

- pages 1 and 2 list results for all analytes reported by at least twice, counting both participants and NIST analysts.

- page 3 lists values for all analytes reported by only once. This page also provides a legend for pages 1 and 2 .

- $\quad$ page 4 summarizes each participants' performance for total retinol, $\alpha$-tocopherol, and total $\beta$-carotene. These summaries are compatible with the percent bias evaluation advice given in the RR20 Report. However, the current bias summaries are estimated relative to the median of all reported values for each analyte in each serum rather than to the "Trimmed Core Lab Average" used in the original and detailed in Appendix J. These original reference values were estimated from on-time results of the more experienced participants, with subjective exclusion of results deemed non-representative.

In the original data sheets for RR20, one participant commented that the two "Serum 141 "vials they received yielded quite discordant results. Only the most concordant of their two sets of results were included in the original Final Report (see Appendix H). When reviewing the data for this report, it was noted that the excluded Serum 141 results from this participant as well as those reported by two other participants matched the results expected for Serum 121 distributed in RR18; the intended Serum 141 material was distributed as Serum 122 in RR18. Thus it is quite plausible that several vials of Serum 121 were incorrectly relabeled and distributed in RR20. For this reason, the updated AllLab Report lists the three sets of discordant Serum 141 results under the heading "Serum 141a". The median values listed for Serum 141a are those for Serum 121 in RR18.

To ensure confidentiality, the laboratory identifiers used in this "All-Lab Report" have been altered from those used in RR20. The only attributed results are those reported by NIST. The NIST results are not used in the assessment of the consensus summary results of the study.

Note: The results designated NISTa and NISTc in this updated All-Lab report are both designated "NIST" in the Tables of Appendix H. 


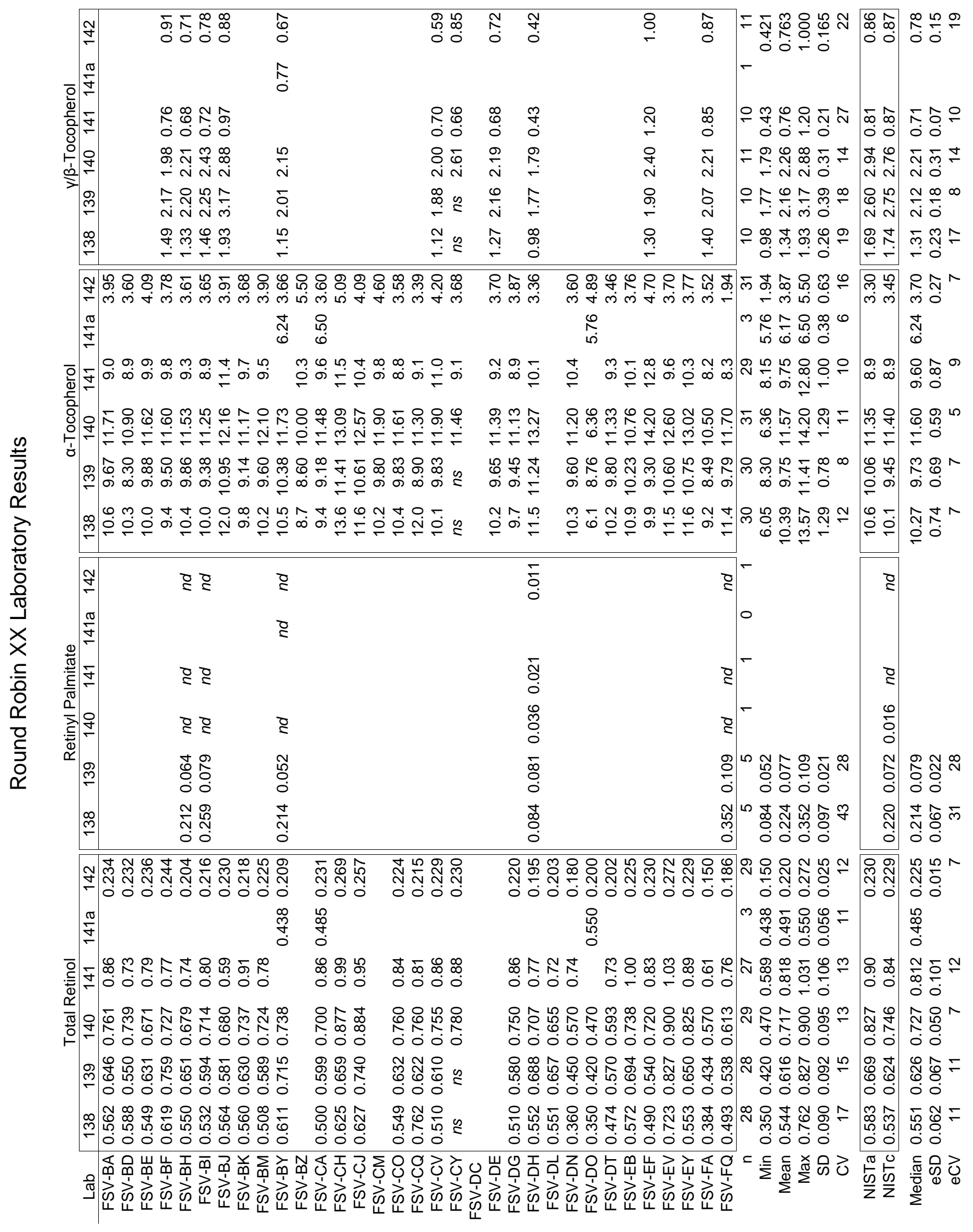




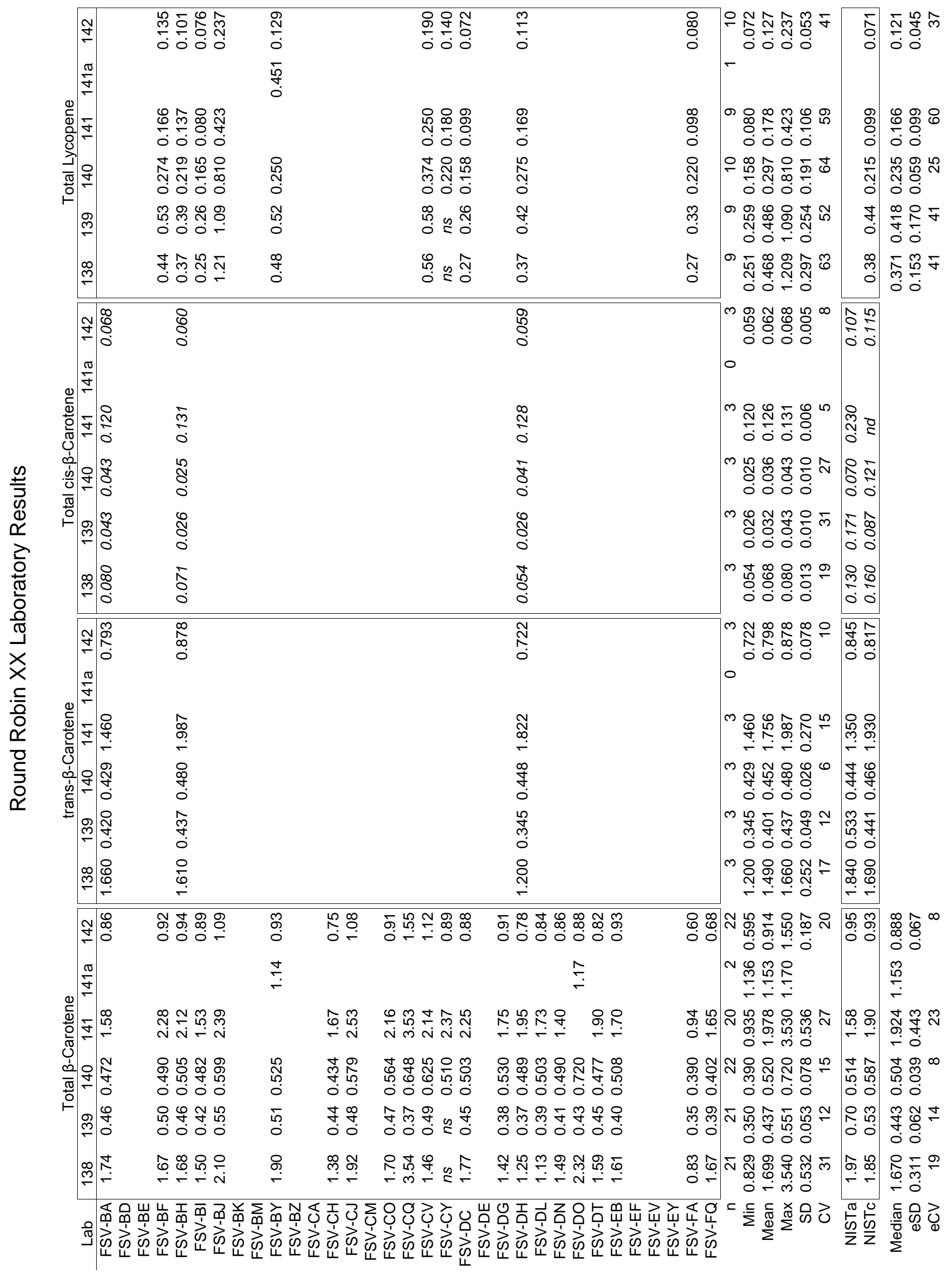




\section{Round Robin XX Laboratory Results}

Analytes Reported By One Laboratory

Values in $\mu \mathrm{g} / \mathrm{mL}$

\begin{tabular}{r|c|cccccc|}
\cline { 2 - 7 } Analyte & Code & 138 & 139 & 140 & 141 & $141 \mathrm{a}$ & 142 \\
\hline Total $\alpha$-Carotene & FSV-BJ & 0.083 & 0.041 & 0.032 & 0.043 & & 0.036
\end{tabular}

\section{Table Legend}

\begin{tabular}{r|l} 
Symbol & \multicolumn{1}{|c}{ Interpretation } \\
\hline$n a$ & Not available (not reported by participant) \\
$n d$ & Not detected (not reported or reported as '0', 'not determined', etc.) \\
$n s$ & No sample (sample either damaged or not sent) \\
$i t a l i c s$ & Value calculated from reported results \\
$n$ & Number of non-NIST laboratories reporting quantitative results \\
Min & Minimum non-NIST reported value. \\
Mean & Average over all non-NIST reported values. \\
Max & Maximum non-NIST reported value. \\
SD & Standard deviation over all non-NIST values. \\
$\mathrm{CV}$ & Coefficient of Variation (\% relative standard deviation): 100*SD/Mean \\
Median & Median over all non-NIST reported values \\
eSD & $\begin{array}{l}\text { Robust estimate of SD based on the adjusted median absolute } \\
\text { difference from the median (MADe) }\end{array}$ \\
eCV & Robust estimate of CV, 100*eSD/Median
\end{tabular}




\section{Round Robin XX Laboratory Results}

\section{\%Bias Summary}

\begin{tabular}{|c|c|c|c|c|c|}
\hline $\mathrm{Lab}$ & TR & aT & $\mathrm{bC}$ & Label & Definition \\
\hline FSV-BA & $4 \pm 1$ & $1 \pm 5$ & $-4 \pm 9$ & Lab & Participant code \\
\hline FSV-BD & $-2 \pm 8$ & $-6 \pm 6$ & & TR & Total Retinol \\
\hline FSV-BE & $-1 \pm 5$ & $3 \pm 5$ & & aT & a-Tocopherol \\
\hline FSV-BF & $7 \pm 11$ & $-1 \pm 4$ & $6 \pm 9$ & bC & Total b-Carotene \\
\hline $\mathrm{FSV}-\mathrm{BH}$ & $-4 \pm 6$ & $-1 \pm 2$ & $4 \pm 4$ & $\%$ Bias & (Mean \pm SD) of individual serum biases \\
\hline FSV-BI & $-3 \pm 1$ & $-4 \pm 2$ & $-8 \pm 8$ & & \\
\hline FSV-BJ & $-7 \pm 12$ & $12 \pm 6$ & $23 \pm 3$ & Mean & Average of $\left(\mathrm{x}_{\mathrm{i}}-\right.$ Median $\left._{\mathrm{i}}\right) /$ Median $_{\mathrm{i}}$ \\
\hline FSV-BK & $2 \pm 6$ & $-3 \pm 3$ & & SD & Standard deviation of $\left(\mathrm{x}_{\mathrm{i}}-\right.$ Median $\left._{\mathrm{i}}\right) /$ Median $_{\mathrm{i}}$ \\
\hline FSV-BM & $-4 \pm 3$ & $1 \pm 3$ & & $x_{i}$ & Result for analyte in serum $i$ \\
\hline FSV-BY & $2 \pm 11$ & $1 \pm 5$ & $9 \pm 5$ & Median $_{\mathrm{i}}$ & Median of non-NIST results in serum ${ }_{i}$ \\
\hline FSV-BZ & & $3 \pm 27$ & & & \\
\hline FSV-CA & $-2 \pm 5$ & $-3 \pm 3$ & & & \\
\hline $\mathrm{FSV}-\mathrm{CH}$ & $16 \pm 7$ & $24 \pm 10$ & $-12 \pm 7$ & \multirow{23}{*}{\multicolumn{2}{|c|}{$\begin{array}{l}\text { The original analysis listed \% Bias for each result for } \\
\text { each serum calculated relative to the trimmed "Avg" of } \\
\text { that analyte in the serum. The summary values } \\
\text { reported here are the (arithmetic mean } \pm \text { standard } \\
\text { deviation) of each laboratory's reported results for the } \\
\text { analyte estimated relative to each serum's median- } \\
\text { based reference value. } \\
\text { For all sera except } 141 a \text {, the reference value is the } \\
\text { median of results in this study. For serum 141a, the } \\
\text { reference value is the median of of results for serum } \\
121 \text { in Round Robin XVIII. }\end{array}$}} \\
\hline FSV-CJ & $17 \pm 3$ & $10 \pm 2$ & $19 \pm 9$ & & \\
\hline FSV-CM & & $6 \pm 10$ & & & \\
\hline FSV-CO & $2 \pm 2$ & $-2 \pm 4$ & $7 \pm 5$ & & \\
\hline FSV-CQ & $8 \pm 18$ & $-2 \pm 11$ & $57 \pm 50$ & & \\
\hline FSV-CV & $0 \pm 5$ & $6 \pm 7$ & $12 \pm 16$ & & \\
\hline FSV-CY & $6 \pm 3$ & $-2 \pm 3$ & $8 \pm 13$ & & \\
\hline FSV-DC & & & $4 \pm 7$ & & \\
\hline FSV-DE & & $-1 \pm 2$ & & & \\
\hline FSV-DG & $-2 \pm 6$ & $-3 \pm 5$ & $-6 \pm 9$ & & \\
\hline FSV-DH & $-2 \pm 8$ & $7 \pm 10$ & $-11 \pm 10$ & & \\
\hline FSV-DL & $-5 \pm 7$ & & $-12 \pm 12$ & & \\
\hline FSV-DN & $-23 \pm 10$ & $0 \pm 5$ & $-10 \pm 10$ & & \\
\hline FSV-DO & $-21 \pm 21$ & $-15 \pm 31$ & $18 \pm 22$ & & \\
\hline FSV-DT & $-12 \pm 4$ & $-2 \pm 3$ & $-4 \pm 4$ & & \\
\hline FSV-EB & $8 \pm 9$ & $2 \pm 6$ & $-4 \pm 7$ & & \\
\hline FSV-EF & $-4 \pm 8$ & $15 \pm 18$ & & & \\
\hline FSV-EV & $27 \pm 5$ & $6 \pm 6$ & & & \\
\hline FSV-EY & $6 \pm 6$ & $9 \pm 4$ & & & \\
\hline FSV-FA & $-28 \pm 5$ & $-11 \pm 4$ & $-36 \pm 15$ & & \\
\hline FSV-FQ & $-13 \pm 4$ & $-10 \pm 23$ & $-14 \pm 9$ & & \\
\hline $\mathrm{NISTa}$ & $8 \pm 4$ & $-3 \pm 7$ & $14 \pm 28$ & & \\
\hline NISTc & $1 \pm 2$ & $-4 \pm 3$ & $10 \pm 8$ & & \\
\hline
\end{tabular}

